\title{
Turbulence dynamics near a turbulent/non-turbulent interface
}

Article

Accepted Version

Teixeira, M. A. C. and da Silva, C. B. (2012) Turbulence dynamics near a turbulent/non-turbulent interface. Journal Of Fluid Mechanics, 695. pp. 257-287. ISSN 0022-1120 doi: https://doi.org/10.1017/jfm.2012.17 Available at https://centaur.reading.ac.uk/29236/

It is advisable to refer to the publisher's version if you intend to cite from the work. See Guidance on citing.

Published version at: http://dx.doi.org/10.1017/jfm.2012.17

To link to this article DOI: http://dx.doi.org/10.1017/jfm.2012.17

Publisher: Cambridge University Press

All outputs in CentAUR are protected by Intellectual Property Rights law, including copyright law. Copyright and IPR is retained by the creators or other copyright holders. Terms and conditions for use of this material are defined in the End User Agreement.

\section{www.reading.ac.uk/centaur}

\section{CentAUR}

Central Archive at the University of Reading

Reading's research outputs online 


\title{
Turbulence dynamics near a turbulent/non-turbulent interface
}

\author{
M. A. C. TEIXEIRA A AND C. B. DA SIL V A ${ }^{2}$ \\ ${ }^{1}$ CGUL/IDL, University of Lisbon, Edifício C8, Campo Grande, 1749-016 Lisbon, Portugal \\ ${ }^{2}$ IDMEC/IST, Technical University of Lisbon, Pav. Mecânica I, $1^{\circ}$ andar/esq./LASEF, Av. \\ Rovisco Pais, 1049-001 Lisbon, Portugal
}

(Received 2 February 2012)

The characteristics of the boundary layer separating a turbulence region from an irrotational (or non-turbulent) flow region are investigated using rapid distortion theory (RDT). The turbulence region is approximated as homogeneous and isotropic far away from the bounding turbulent/non-turbulent (T/NT) interface, which is assumed to remain approximately flat. Inviscid effects resulting from the continuity of the normal velocity and pressure at the interface, in addition to viscous effects resulting from the continuity of the tangential velocity and shear stress, are taken into account by considering a sudden insertion of the T/NT interface, in the absence of mean shear. Profiles of the velocity variances, turbulent kinetic energy (TKE), viscous dissipation rate $(\varepsilon)$, turbulence length scales, and pressure statistics are derived, showing an excellent agreement with results from Direct Numerical Simulations. Interestingly, the normalized inviscid flow statistics at the T/NT interface do not depend on the form of the assumed TKE spectrum. Outside the turbulent region, where the flow is irrotational (except inside a thin viscous boundary layer), $\varepsilon$ decays as $z^{-6}$, where $z$ is the distance from the T/NT interface. The mean pressure distribution is calculated using RDT, and exhibits a decrease towards the turbulence region due to the associated velocity fluctuations, consistent with the generation of a mean entrainment velocity. The vorticity variance and $\varepsilon$ display large maxima at the T/NT interface due to the inviscid discontinuities of the tangential velocity variances existing there, and these maxima are quantitatively related to the thickness $\delta$ of the viscous boundary layer (VBL). For an equilibrium VBL, the RDT analysis suggests that $\delta \sim \eta$ (where $\eta$ is the Kolmogorov microscale), which is consistent with the scaling law identified in a very recent DNS study for shear-free T/NT interfaces.

Key Words: Isotropic turbulence, Turbulence theory, Turbulence modelling

\section{Introduction}

Interfaces separating turbulent from non-turbulent fluid are ubiquitous in geophysical and industrial flows, e.g. at the edges of jets, plumes, mixing layers and wakes. In the atmosphere or in the ocean, such interfaces delimit the planetary boundary layers (PBLs) in each fluid. Entrainment, by which turbulent regions expand through 'ingestion' of external irrotational fluid, take place across these interfaces. Entrainment is an extraordinarily important process, controlling exchanges of mass, momentum, heat and contaminants, and the rate at which turbulent regions grow, i.e. the spreading rate of plumes or the deepening of PBLs (Turner 1986).

Entrainment has initially been thought to be driven by 'engulfing' motions induced by 
the large-scale turbulence structures (Townsend 1966, 1976). This has influenced, among other aspects, the development of entrainment assumptions in plumes, which typically rely on integral properties of the flow, such as the mean along-plume velocity (Turner 1986; Teixeira \& Miranda 1997). However, more recent investigations (Westerweel et al. 2005, 2009) have drawn attention to the importance of small-scale 'nibbling' motions (Corrsin \& Kistler 1955) in the entrainment process, which are intrinsically connected with the viscous coupling between the turbulent and non-turbulent regions. According to this model, the entrainment is caused by small-scale eddy motions, acting to diffuse vorticity outwards from the turbulence into the irrotational flow region. Nevertheless, it is still largely accepted that the entrainment rate is imposed by the large-scale eddy motions.

The small-scale physics of the flow near turbulent/non-turbulent (T/NT) interfaces is important also in the development of subgrid-scale models for Large-Eddy Simulations (LES). As shown recently by da Silva (2009), some of the classical assumptions behind these parametrizations, e.g. local equilibrium, negligible kinetic energy at the sub-grid scale level, are not satisfied near T/NT interfaces (for example, at the edge of a plume), and this makes the performance of LES models unsatisfactory, compromising the correct prediction of the mixing rates near the $\mathrm{T} / \mathrm{NT}$ interfaces. In order to be able to improve sub-grid scale models in this context, it is necessary to understand the small-scale details of the flow.

The techniques of conditional sampling, applied to Direct Numerical Simulation (DNS) or to experimental data of turbulent flows, have enabled the elaboration of a much more detailed conceptual picture of the T/NT interface (Westerweel et al. 2005, 2009). The turbulent kinetic energy (TKE) budget has been analyzed, and even in relatively complex flows with mean shear such as jets, some distinctive features have been identified. In particular, the T/NT interface was found to be characterized by a sharp vorticity jump, with thickness of the order of the Taylor microscale, between a region where the turbulent vorticity is high and more or less homogeneous and isotropic, and a region of approximately irrotational flow (Westerweel et al. 2005, 2009; da Silva \& Pereira 2008). These features suggest the use of Rapid Distortion Theory (RDT), with its idealized symmetry assumptions and simplified geometry, as a potentially fruitful method to understand these flows. RDT is able to model both inviscid and viscous linear processes in turbulent flows, including the effect of boundaries. In this technique the turbulence is assumed to remain frozen, and boundaries are treated as suddenly-inserted, both assumptions being formally valid for times much shorter than one eddy-turn over time.

Phillips (1955) gave one of the pioneering contributions to the study of the statistics of the irrotational velocity fluctuations outside a turbulent region. More recently, Carruthers \& Hunt (1986) used RDT to address the problem of turbulence near a stably stratified, non-turbulent region, corresponding to a simplified model of the edge of the PBL. A particular case of interest to the more basic problem of $\mathrm{T} / \mathrm{NT}$ interfaces without stratification can be derived from the results of Carruthers \& Hunt (1986) by setting the static stability in the non-turbulent region to zero. However, these authors did not explicitly address the effect of the viscous boundary layer (VBL), nor did they calculate statistics such as the dissipation rate.

These considerations motivate the present study, where RDT is used to calculate flow statistics across a T/NT interface in a constant-density fluid. In the present work the simplest idealized conditions are considered, with initially quiescent flow in half of the domain, and initially homogeneous and isotropic shear-free (i.e. with zero mean shear) turbulence in the other half. It is assumed that at the initial time a T/NT interface is suddenly inserted, imposing boundary conditions between the two regions of the flow. 
This leads to the appearance of irrotational velocity corrections, and the growth of VBLs to both sides of the interface. Although in real flows the T/NT interface is quite convoluted and propagates into the non-turbulent region, here it is treated as essentially flat and fixed for simplicity. Additionally, although in this situation appreciable entrainment velocities develop, that aspect is not explicitly addressed here.

In the present work, Reynolds stresses and dissipation rates are calculated from the theoretical RDT model, in order to elucidate the physical mechanisms of the adjustment between the turbulent and non-turbulent regions of the flow. The inviscid values of these statistics at either side of the T/NT interface are found not to depend on the energy spectrum of the turbulence, being expressed in terms of their values in the bulk of the turbulent flow. The asymptotic behaviour of the flow statistics in the irrotational nonturbulent region is also analyzed. New theoretical calculations developed here, taking the VBLs into account, are compared with DNS performed at relatively low Reynolds number. Finally, physical arguments based on RDT are advanced for justifying possible scalings put forward by previous authors for the equilibrium thickness of the T/NT interface.

This paper is organized as follows. In $\S 2$, the theoretical model is presented, and the setup of the numerical simulations is described. Section 3 contains the main results, namely profiles (across the T/NT interface) of various turbulence statistics obtained from RDT, and their comparisons with DNS. Finally, in $\S 4$, the main findings of this study are summarized.

\section{Theoretical model}

The theoretical approach employed here is akin to that developed by Teixeira \& Belcher (2000) for shear-free turbulence bounded by solid or free surfaces. At time $t=0 \mathrm{a} \mathrm{T} / \mathrm{NT}$ interface is imposed at $z=0$ of a Cartesian coordinate system, separating the turbulent zone of a fluid at $z>0$ from a quiescent zone at $z<0$. The $z$ coordinate is therefore perpendicular to the T/NT interface (pointing towards the turbulence), whereas $x$ and $y$ are parallel to the interface (see figure 1 ). At $t>0$, the flow is forced to adjust to the boundary conditions at $z=0$, leading to the appearance of both inviscid and viscous velocity corrections at either side of the interface. This leads the velocity field in the turbulent and non-turbulent parts of flow to be expressed, respectively, as

$$
\begin{aligned}
& \boldsymbol{u}_{+}=\boldsymbol{u}^{(H)}+\boldsymbol{u}_{+}^{(S)}+\boldsymbol{u}_{+}^{(V)}, \quad z>0, \\
& \boldsymbol{u}_{-}=\boldsymbol{u}_{-}^{(S)}+\boldsymbol{u}_{-}^{(V)}, \quad z<0,
\end{aligned}
$$

where $\boldsymbol{u}_{ \pm}(x, y, z, t)$ is the perturbation velocity field (turbulent or not), which has zero average (no mean flow is assumed to exist). Here, and from now onwards, use of the index \pm purports to refer more compactly to variables in both regions $z>0$ and $z<0$. In (2.1) the flow comprises (for $z>0$ ) a term corresponding to homogeneous and isotropic turbulence (hereafter HIT), $\boldsymbol{u}^{(H)}$ (which describes the far-field turbulent zone and is assumed to remain frozen and undistorted), irrotational velocity corrections $\boldsymbol{u}_{ \pm}^{(S)}=\boldsymbol{\nabla} \phi_{ \pm}^{(S)}$ deriving from velocity potentials $\phi_{ \pm}^{(S)}$, which exist primarily inside 'blocking layers' at each side of $z=0$, and viscous corrections $\boldsymbol{u}_{ \pm}^{(V)}$, which only exist inside the VBLs directly adjacent to $z=0$. All these flow components are necessary to satisfy the continuity of the total velocity and stress at $z=0$. Although the T/NT interface, even if initially flat, is in reality advected by the turbulent flow and becomes progressively deformed, this nonlinear effect is neglected in the model. Thus the T/NT interface, where the boundary conditions are applied, is assumed to remain always at $z=0$. 


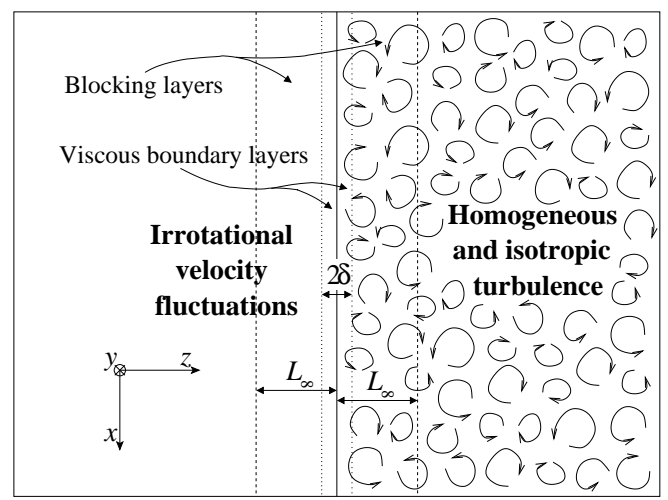

FIGURE 1. Schematic diagram showing the idealized situation considered in the present model. $L_{\infty}$ is the integral length scale of the turbulence and $\delta$ is the VBL thickness.

The equations of motion valid for a fluid with constant density are:

$$
\begin{aligned}
\frac{\partial \boldsymbol{u}}{\partial t}+(\boldsymbol{u} \cdot \boldsymbol{\nabla}) \boldsymbol{u} & =-\frac{1}{\rho} \boldsymbol{\nabla} p+\nu \nabla^{2} \boldsymbol{u}, \\
\boldsymbol{\nabla} \cdot \boldsymbol{u} & =0,
\end{aligned}
$$

where $\boldsymbol{u}=(u, v, w)$ may be either $\boldsymbol{u}_{+}$or $\boldsymbol{u}_{-}$, defined above, $p$ is the pressure perturbation, $\rho$ is the density and $\nu$ is the kinematic viscosity of the fluid. Equation (2.2) does not include the gravity because this is assumed to be balanced by the mean vertical pressure gradient via hydrostatic equilibrium. By taking the curl of (2.2), a vorticity equation may be derived:

$$
\frac{\partial \boldsymbol{\omega}}{\partial t}+(\boldsymbol{u} \cdot \boldsymbol{\nabla}) \boldsymbol{\omega}=(\boldsymbol{\omega} \cdot \boldsymbol{\nabla}) \boldsymbol{u}+\nu \nabla^{2} \boldsymbol{\omega}
$$

where $\boldsymbol{\omega}=\boldsymbol{\nabla} \times \boldsymbol{u}$ is the vorticity vector.

It can be shown through scaling of (2.4) outside the VBL that the first term on the lefthand side dominates for sufficiently short timescales. Therefore, for the sudden insertion of a T/NT interface, and in accordance with (2.1), the velocity corrections outside the VBLs are necessarily irrotational, since the turbulent vorticity $\boldsymbol{\omega}$ does not change. This is a good approximation for $t \ll T_{L}$, where $T_{L}=l / q$ is the eddy turn-over time of the turbulence, defined in terms of a characteristic turbulence length scale $l$ and velocity scale $q$. In the above scaling it was assumed that the inviscid velocity scales on $q$, and varies over a distance of order $l$. Mass conservation for $\boldsymbol{u}^{(H)}$ is assumed by default, so the same conservation law applied to $\boldsymbol{u}_{ \pm}^{(S)}$ yields

$$
\nabla^{2} \phi_{+}^{(S)}=0, \quad \nabla^{2} \phi_{-}^{(S)}=0 .
$$

These equations must be solved subject to the inviscid boundary conditions, which state that the irrotational velocity corrections must tend to zero at infinity, and both the normal velocity $w$ and the pressure $p$ must be continuous at the T/NT interface. In terms of the velocity potentials, this means (cf. Carruthers \& Hunt 1986):

$$
\begin{aligned}
\phi_{+}^{(S)}(z \rightarrow+\infty) & =0, \quad \phi_{-}^{(S)}(z \rightarrow-\infty)=0, \\
w^{(H)}(z=0)+\frac{\partial \phi_{+}^{(S)}}{\partial z}(z=0) & =\frac{\partial \phi_{-}^{(S)}}{\partial z}(z=0), \quad \phi_{+}^{(S)}(z=0)=\phi_{-}^{(S)}(z=0) .
\end{aligned}
$$

Inside the VBLs, the viscous velocity corrections that are necessary to satisfy the boundary conditions vary normal to the $\mathrm{T} / \mathrm{NT}$ interface over a distance of order $\delta$, the 
VBL thickness. This makes the corresponding normal velocity components be smaller than the tangential ones by a factor of $\delta / l$. Taking these aspects into account, scale analysis of (2.2) shows that inside the VBLs and subject to the same condition $t \ll T_{L}$, this equation approximately reduces to

$$
\frac{\partial \boldsymbol{v}_{+}^{(V)}}{\partial t}=\nu \frac{\partial^{2} \boldsymbol{v}_{+}^{(V)}}{\partial z^{2}}, \quad \frac{\partial \boldsymbol{v}_{-}^{(V)}}{\partial t}=\nu \frac{\partial^{2} \boldsymbol{v}_{-}^{(V)}}{\partial z^{2}},
$$

where $\boldsymbol{v}_{ \pm}^{(V)}=\left(u_{ \pm}^{(V)}, v_{ \pm}^{(V)}, 0\right)$ are the viscous velocity corrections tangential to the $\mathrm{T} / \mathrm{NT}$ interface. The boundary conditions applied to these equations are the following. The viscous velocity corrections must decay to zero at infinity, and both the total tangential velocity and its normal derivative (proportional to the viscous shear stress) must be continuous at the T/NT interface, i.e.:

$$
\begin{gathered}
\boldsymbol{v}_{+}^{(V)}(z \rightarrow+\infty)=0, \quad \boldsymbol{v}_{-}^{(V)}(z \rightarrow-\infty)=0 \\
\boldsymbol{v}^{(H)}(z=0)+\boldsymbol{v}_{+}^{(S)}(z=0)+\boldsymbol{v}_{+}^{(V)}(z=0)=\boldsymbol{v}_{-}^{(S)}(z=0)+\boldsymbol{v}_{-}^{(V)}(z=0), \\
\frac{\partial \boldsymbol{v}^{(H)}}{\partial z}(z=0)+\frac{\partial \boldsymbol{v}_{+}^{(S)}}{\partial z}(z=0)+\frac{\partial \boldsymbol{v}_{+}^{(V)}}{\partial z}(z=0)=\frac{\partial \boldsymbol{v}_{-}^{(S)}}{\partial z}(z=0)+\frac{\partial \boldsymbol{v}_{-}^{(V)}}{\partial z}(z=0),
\end{gathered}
$$

where $\boldsymbol{v}^{(H)}=\left(u^{(H)}, v^{(H)}, 0\right)$ and $\boldsymbol{v}_{ \pm}^{(S)}=\left(u_{ \pm}^{(S)}, v_{ \pm}^{(S)}, 0\right)$ are, respectively, the HIT velocity and the irrotational velocity corrections tangential to the T/NT interface.

In fact, because (2.8) are equations only for the tangential velocity components, use of the continuity equation for the viscous velocity corrections,

$$
\nabla \cdot \boldsymbol{u}_{ \pm}^{(V)}=0
$$

shows that the horizontal inhomogeneity of $\boldsymbol{v}_{ \pm}^{(V)}$ induces a non-zero normal velocity $w_{ \pm}^{(V)}$ of order $q(\delta / l)$ that extends outside the VBLs. This makes it necessary to add a correction of similar magnitude to $\boldsymbol{u}_{ \pm}^{(S)}$ to satisfy the boundary conditions, which in turn will induce a viscous normal velocity of $O\left[q(\delta / l)^{2}\right]$, and so on. As in Teixeira \& Belcher (2000), this leads to a power series expansion for the solutions, namely:

$$
\begin{aligned}
& \phi_{ \pm}^{(S)}=\phi_{ \pm}^{(S 0)}+\left(\frac{\delta}{l}\right) \phi_{ \pm}^{(S 1)}+\left(\frac{\delta}{l}\right)^{2} \phi_{ \pm}^{(S 2)}+\ldots \\
& \boldsymbol{u}_{ \pm}^{(V)}=\boldsymbol{u}_{ \pm}^{(V 0)}+\left(\frac{\delta}{l}\right) \boldsymbol{u}_{ \pm}^{(V 1)}+\left(\frac{\delta}{l}\right)^{2} \boldsymbol{u}_{ \pm}^{(V 2)}+\ldots
\end{aligned}
$$

Since this expansion arises due to a VBL effect, in the inviscid approximation only $\phi_{ \pm}^{(S 0)}=\phi_{ \pm}^{(S)}$ are non-zero in $(2.12)$.

\subsection{Fourier analysis}

Consistent with the geometry of the problem (see figure 1) and the boundary conditions (2.6)-(2.7) and (2.9)-(2.10), the flow is assumed to be statistically homogeneous and unlimited along the $x$ and $y$ directions. Since the velocity field $\boldsymbol{u}^{(H)}$ corresponds to HIT, it is also statistically homogeneous along the $z$ direction. Thus it can be expressed as a 3D Fourier integral:

$$
\boldsymbol{u}^{(H)}(\boldsymbol{x})=\iiint \hat{\boldsymbol{u}}^{(H)}(\boldsymbol{k}) \mathrm{e}^{\mathrm{i} \boldsymbol{k} \cdot \boldsymbol{x}} \mathrm{d} k_{1} \mathrm{~d} k_{2} \mathrm{~d} k_{3},
$$


where $\hat{\boldsymbol{u}}^{(H)}$ is the corresponding Fourier transform, $\boldsymbol{x}=(x, y, z)$ and $\boldsymbol{k}=\left(k_{1}, k_{2}, k_{3}\right)$ is the wavenumber vector. The velocity potentials and the viscous velocity corrections are inhomogeneous along $z$, since they only exist near the T/NT interface. Therefore, they, and the total velocity, may be expressed instead as 2D Fourier integrals:

$$
\begin{aligned}
\phi_{ \pm}^{(S)}(\boldsymbol{x}, t) & =\iint \hat{\phi}_{ \pm}^{(S)}\left(k_{1}, k_{2}, z, t\right) \mathrm{e}^{\mathrm{i}\left(k_{1} x+k_{2} y\right)} \mathrm{d} k_{1} \mathrm{~d} k_{2}, \\
\boldsymbol{u}_{ \pm}^{(V)}(\boldsymbol{x}, t) & =\iint \hat{\boldsymbol{u}}_{ \pm}^{(V)}\left(k_{1}, k_{2}, z, t\right) \mathrm{e}^{\mathrm{i}\left(k_{1} x+k_{2} y\right)} \mathrm{d} k_{1} \mathrm{~d} k_{2} \\
\boldsymbol{u}_{ \pm}(\boldsymbol{x}, t) & =\iint \hat{\boldsymbol{u}}_{ \pm}\left(k_{1}, k_{2}, z, t\right) \mathrm{e}^{\mathrm{i}\left(k_{1} x+k_{2} y\right)} \mathrm{d} k_{1} \mathrm{~d} k_{2}
\end{aligned}
$$

where $\hat{\phi}_{ \pm}^{(S)}, \hat{\boldsymbol{u}}_{ \pm}^{(V)}$ and $\hat{\boldsymbol{u}}_{ \pm}$are the corresponding Fourier transforms. When these expressions are inserted into (2.5) and (2.8), these equations may be solved for $\hat{\phi}_{ \pm}^{(S)}$ and $\hat{\boldsymbol{v}}_{ \pm}^{(V)}$ (which also take the form of power series of $\delta / l$ ) at the required order. With the boundary conditions (2.6)-(2.7) and (2.9)-(2.10), the arbitrary constants that appear in $\hat{\phi}_{ \pm}^{(S)}$ and $\hat{\boldsymbol{u}}_{ \pm}^{(V)}$ may be eliminated and a complete solution to the problem can be found.

\subsubsection{Inviscid solutions}

If the VBLs are disregarded, it can be shown (cf. Carruthers \& Hunt 1986) that

$$
\begin{aligned}
& \hat{\phi}_{+}^{(S)}=\frac{1}{2} \int \hat{w}^{(H)} \frac{\mathrm{e}^{-k_{12} z}}{k_{12}} \mathrm{~d} k_{3}, \quad z>0, \\
& \hat{\phi}_{-}^{(S)}=\frac{1}{2} \int \hat{w}^{(H)} \frac{\mathrm{e}^{k_{12} z}}{k_{12}} \mathrm{~d} k_{3}, \quad z<0,
\end{aligned}
$$

where $k_{12}=\left(k_{1}^{2}+k_{2}^{2}\right)^{1 / 2}$. Taking into account the fact that $\hat{\boldsymbol{v}}_{ \pm}^{(S)}=\left(\mathrm{i} k_{1} \hat{\phi}_{ \pm}^{(S)}, \mathrm{i} k_{2} \hat{\phi}_{ \pm}^{(S)}\right)$ and $\hat{w}_{ \pm}^{(S)}=\partial \hat{\phi}_{ \pm}^{(S)} / \partial z$, the $2 \mathrm{D}$ Fourier transforms of the total velocity in the turbulent and non-turbulent zones, respectively, can be written

$$
\begin{aligned}
& \hat{u}_{+}=\int\left(\hat{u}^{(H)} \mathrm{e}^{\mathrm{i} k_{3} z}+\hat{w}^{(H)} \frac{\mathrm{i} k_{1}}{2 k_{12}} \mathrm{e}^{-k_{12} z}\right) \mathrm{d} k_{3}, \\
& \hat{v}_{+}=\int\left(\hat{v}^{(H)} \mathrm{e}^{\mathrm{i} k_{3} z}+\hat{w}^{(H)} \frac{\mathrm{i} k_{2}}{2 k_{12}} \mathrm{e}^{-k_{12} z}\right) \mathrm{d} k_{3}, \\
& \hat{w}_{+}=\int \hat{w}^{(H)}\left(\mathrm{e}^{\mathrm{i} k_{3} z}-\frac{1}{2} \mathrm{e}^{-k_{12} z}\right) \mathrm{d} k_{3}, \quad z>0, \\
& \hat{u}_{-}=\frac{1}{2} \int \hat{w}^{(H)} \frac{\mathrm{i} k_{1}}{k_{12}} \mathrm{e}^{k_{12} z} \mathrm{~d} k_{3}, \\
& \hat{v}_{-}=\frac{1}{2} \int \hat{w}^{(H)} \frac{\mathrm{i} k_{2}}{k_{12}} \mathrm{e}^{k_{12} z} \mathrm{~d} k_{3}, \\
& \hat{w}_{-}=\frac{1}{2} \int \hat{w}^{(H)} \mathrm{e}^{k_{12} z} \mathrm{~d} k_{3}, \quad z<0 .
\end{aligned}
$$

These solutions are similar to those derived by Carruthers \& Hunt (1986) in the limit of no stratification.

\subsubsection{Viscous solutions}

If the VBLs are taken into account, the power series procedure (2.12) must be followed. Then (2.15)-(2.16) become expressions for $\hat{\phi}_{ \pm}^{(S 0)}$. In fact, the series expansions are only 
calculated here up to zeroth-order for $\phi_{ \pm}^{(S)}$, to first-order for $\boldsymbol{v}_{ \pm}^{(V)}$ and to second-order in $\delta / l$ for $w_{ \pm}^{(V)}$. This is done to keep consistency with both conservation of mass and the boundary conditions at the T/NT interface. However, the truncation of the series makes it impossible to strictly satisfy all boundary conditions in the problem. As in Teixeira \& Belcher (2000), it is chosen here to slightly violate the boundary conditions in the far field of the turbulence zone, because this has little impact on the statistics to be calculated. This implies a departure of the velocity field from its consistent value deep inside the turbulence zone by an amount of the same order as the neglected terms in the power series. Given that $\delta / l$ is expected to be small, this is not a very serious limitation. The only way to alleviate this problem would be by considering a larger number of terms in the series expansion. But this would have limited physical relevance, because above a certain order the terms considered are necessarily smaller than the nonlinear terms neglected by the RDT assumptions.

The viscous velocity corrections can be written:

$$
\begin{aligned}
\hat{u}_{+}^{(V)} & =\frac{1}{2} \int\left[\left(1-\frac{1}{2} \mathrm{i} \sqrt{\pi} \delta k_{3}\right) \hat{u}^{(H)}+\frac{1}{2} \mathrm{i} \sqrt{\pi} \delta k_{1} \hat{w}^{(H)}\right] \mathrm{d} k_{3}\left[\operatorname{erf}\left(\frac{z}{\delta}\right)-1\right] \\
\hat{v}_{+}^{(V)} & =\frac{1}{2} \int\left[\left(1-\frac{1}{2} \mathrm{i} \sqrt{\pi} \delta k_{3}\right) \hat{v}^{(H)}+\frac{1}{2} \mathrm{i} \sqrt{\pi} \delta k_{2} \hat{w}^{(H)}\right] \mathrm{d} k_{3}\left[\operatorname{erf}\left(\frac{z}{\delta}\right)-1\right] \\
\hat{w}_{+}^{(V)} & =\frac{1}{2} \int \hat{w}^{(H)}\left\{\delta\left(\mathrm{i} k_{3}+\frac{1}{2} \sqrt{\pi} \delta k^{2}\right)\left[\frac{z}{\delta}\left(\operatorname{erf}\left(\frac{z}{\delta}\right)-1\right)+\frac{\mathrm{e}^{-z^{2} / \delta^{2}}}{\sqrt{\pi}}\right]-\delta^{2} k^{2}\right\} \mathrm{d} k_{3}, \\
z>0, & \\
\hat{u}_{-}^{(V)} & =\frac{1}{2} \int\left[\left(1+\frac{1}{2} \mathrm{i} \sqrt{\pi} \delta k_{3}\right) \hat{u}^{(H)}-\frac{1}{2} \mathrm{i} \sqrt{\pi} \delta k_{1} \hat{w}^{(H)}\right] \mathrm{d} k_{3}\left[\operatorname{erf}\left(\frac{z}{\delta}\right)+1\right] \\
\hat{v}_{-}^{(V)} & =\frac{1}{2} \int\left[\left(1+\frac{1}{2} \mathrm{i} \sqrt{\pi} \delta k_{3}\right) \hat{v}^{(H)}-\frac{1}{2} \mathrm{i} \sqrt{\pi} \delta k_{2} \hat{w}^{(H)}\right] \mathrm{d} k_{3}\left[\operatorname{erf}\left(\frac{z}{\delta}\right)+1\right] \\
\hat{w}_{-}^{(V)} & =\frac{1}{2} \int \hat{w}^{(H)} \delta\left(\mathrm{i} k_{3}-\frac{1}{2} \sqrt{\pi} \delta k^{2}\right) \mathrm{d} k_{3}\left[\frac{z}{\delta}\left(\operatorname{erf}\left(\frac{z}{\delta}\right)+1\right)+\frac{\mathrm{e}^{-z^{2} / \delta^{2}}}{\sqrt{\pi}}\right] \\
z<0, &
\end{aligned}
$$

where $k=\left(k_{1}^{2}+k_{2}^{2}+k_{3}^{2}\right)^{1 / 2}$ and $\delta=2(\nu t)^{1 / 2}$. In order to obtain the 2D Fourier transform of the total velocity field, (2.17)-(2.18) and (2.19)-(2.20) must be added.

Although in the power series (2.12) $l$ is assumed by Teixeira \& Belcher (2000) to be the integral length scale of the turbulence, this is not evident in (2.19)-(2.20). In fact, whether this scaling is correct or not depends on the form of the energy spectrum of the turbulence, because the turbulent velocity gradients are determined by the highwavenumber tail of this spectrum. This aspect will be addressed next.

\subsection{Turbulence statistics}

As (2.17)-(2.18) and (2.19)-(2.20) suggest, the Fourier transform of the total velocity field may always be expressed in the form

$$
\hat{u}_{i \pm}\left(k_{1}, k_{2}, z, t\right)=\int M_{i j}^{ \pm}\left(k_{1}, k_{2}, k_{3}, z, t\right) \hat{u}_{j}^{(H)}(\boldsymbol{k}) \mathrm{d} k_{3}, \quad i=1,2,3,
$$

where $\left(\hat{u}_{1 \pm}, \hat{u}_{2 \pm}, \hat{u}_{3 \pm}\right)=\left(\hat{u}_{ \pm}, \hat{v}_{ \pm}, \hat{w}_{ \pm}\right)$and $M_{i j}^{ \pm}$are the matrices describing the relationship between the total velocity at each side of the T/NT interface and the HIT velocity existing at $z \rightarrow+\infty$. These matrices are specified in the Appendix. In (2.21) and in the 
following equations, Einstein's index-repetition convention is implied, unless otherwise stated.

The Fourier transforms of the homogeneous and isotropic velocity are related to the corresponding three-dimensional wavenumber spectrum $\Phi_{i j}^{(H)}$ through

$$
\overline{\hat{u}_{i}^{(H) *}(\boldsymbol{k}) \hat{u}_{j}^{(H)}\left(\boldsymbol{k}^{\prime}\right)}=\Phi_{i j}^{(H)}(\boldsymbol{k}) \delta\left(\boldsymbol{k}-\boldsymbol{k}^{\prime}\right)
$$

where the overbar denotes ensemble averaging, the asterisk denotes complex conjugate and $\delta(\boldsymbol{k})$ is the Dirac delta. Taking (2.21) and (2.22) into account, it can be shown that the Reynolds stresses (one-point covariances of the flow velocity) may be expressed in terms of the HIT spectrum in the following way:

$$
\overline{u_{i \pm} u_{j \pm}}=\iiint M_{i k}^{ \pm *} M_{j l}^{ \pm} \Phi_{k l}^{(H)} \mathrm{d} k_{1} \mathrm{~d} k_{2} \mathrm{~d} k_{3} .
$$

Other flow statistics, like the viscous dissipation rate of TKE, depend on the covariances of the velocity gradients, which can be written:

$$
\begin{aligned}
& \overline{\frac{\partial u_{i \pm}}{\partial x_{m}} \frac{\partial u_{j \pm}}{\partial x_{n}}}=\iiint k_{m} k_{n} M_{i k}^{ \pm *} M_{j l}^{ \pm} \Phi_{k l}^{(H)} \mathrm{d} k_{1} \mathrm{~d} k_{2} \mathrm{~d} k_{3} \quad \text { if } \quad m, n=1,2, \\
& \overline{\frac{\partial u_{i \pm}}{\partial x_{m}} \frac{\partial u_{j \pm}}{\partial z}}=-\iiint \mathrm{i} k_{m} M_{i k}^{ \pm *} \frac{\partial M_{j l}^{ \pm}}{\partial z} \Phi_{k l}^{(H)} \mathrm{d} k_{1} \mathrm{~d} k_{2} \mathrm{~d} k_{3} \quad \text { if } \quad m=1,2, \\
& \overline{\frac{\partial u_{i \pm}}{\partial z} \frac{\partial u_{j \pm}}{\partial z}}=\iiint \frac{\partial M_{i k}^{ \pm *}}{\partial z} \frac{\partial M_{j l}^{ \pm}}{\partial z} \Phi_{k l}^{(H)} \mathrm{d} k_{1} \mathrm{~d} k_{2} \mathrm{~d} k_{3} \text {. }
\end{aligned}
$$

Both (2.23) and (2.24) satisfy the required realizability conditions to which such tensors are subjected, since these equations result from the (linear) dynamics of the flow and are thus physically consistent. (For example, $\overline{u_{i \pm} u_{j \pm}}$ is obviously a symmetric tensor that is positive when $i=j$ ).

For isotropic turbulence, the $3 \mathrm{D}$ wavenumber spectrum is related to the energy spectrum $E(k)$ by the expression

$$
\Phi_{i j}^{(H)}=\left(\delta_{i j}-\frac{k_{i} k_{j}}{k^{2}}\right) \frac{E(k)}{4 \pi k^{2}},
$$

where $\delta_{i j}$ is the Kronecker delta. As shown in Teixeira \& Belcher (2000), the definition of the energy spectrum is a non-trivial matter. Those authors used a spectrum which depends on the Reynolds number of the turbulence $R e=q l / \nu$. Here comparison with DNS data at relatively low $R e$ will be carried out, so a form of the spectrum appropriate to those conditions is chosen:

$$
E(k)=\frac{q^{2} l}{(2 \pi)^{1 / 2}}(k l)^{4} \mathrm{e}^{-\frac{1}{2}(k l)^{2}} .
$$

This spectrum, which is akin to one of those suggested by Townsend (1976), only has two free parameters $(q$ and $l$ ). It has the realistic feature of decaying sufficiently fast as $k \rightarrow+\infty$, allowing the calculation of statistics of the velocity gradients, like (2.24). Otherwise, it is adopted for the same reasons as invoked by Hunt, Stretch \& Belcher (2011). $E(k)$ is defined in such a way that

$$
\int_{0}^{+\infty} E(k) \mathrm{d} k=\frac{3}{2} q^{2} \quad \text { and } \quad \varepsilon_{\infty}=2 \nu \int_{0}^{+\infty} k^{2} E(k) \mathrm{d} k=15 \nu \frac{q^{2}}{l^{2}} .
$$

The first integral expresses the TKE in the HIT zone, defining $q$ as the root-mean square 
(RMS) velocity of the turbulence. The second integral expresses the dissipation rate in the bulk of the turbulence $\varepsilon_{\infty}$, and defines $l$ as the Taylor microscale in the same region, i.e. $l=\lambda_{\infty}$ (see Tennekes \& Lumley 1972). Since this energy spectrum is independent of the Reynolds number, it must be strictly applicable in rather limited conditions. In fact, it can be shown that the longitudinal integral length scale in the far-field turbulence implicit in $(2.26)$ is

$$
L_{\infty}=\frac{\pi}{2 q^{2}} \int_{0}^{+\infty} k^{-1} E(k) \mathrm{d} k=\left(\frac{\pi}{2}\right)^{1 / 2} \lambda_{\infty} .
$$

This means that $L_{\infty}$ is related to $\lambda_{\infty}$ by a fixed factor, whose magnitude is of $O(1)$. While this must occur at some relatively small value of $R e$, it is obviously not valid in general.

The fact that in this model $E(k)$ is defined in terms of $\lambda_{\infty}$ will be used henceforth to scale all distances by $\lambda_{\infty}$. Additionally, the Taylor microscale Reynolds number $R e_{\lambda}=q \lambda_{\infty} / \nu$ is henceforth adopted as the relevant $R e$. This is particularly appropriate for comparisons with DNS results, since $\lambda_{\infty}$ is determined with great precision in such numerical simulations. On the other hand, the above considerations make it clear that the power series expansion (2.12) is in fact formulated in terms of $\delta / \lambda_{\infty}$, an aspect that will be touched upon again in $\S 3$.

\subsection{DNS of shear-free turbulence}

DNS of shear-free turbulence (hereafter SFT) were carried out in order to assess the predictions of the RDT model described above. The simulations use a classical NavierStokes solver with pseudo-spectral methods for spatial discretization and a 3rd-order, 3-step Runge-Kutta scheme for temporal advancement. The same code was used in e.g. da Silva \& Taveira (2010). In these simulations, an irrotational/isotropic SFT boundary is generated by instantaneously inserting a velocity field from a previously run DNS of (forced) HIT into the middle of a field of zero initial velocity. As time progresses, the initial HIT region spreads into the irrotational region in the absence of mean shear, thus generating a flow of SFT.

The simulation starts with classical (forced) HIT in a periodic box with sides equal to $\left(L_{x} \times L_{y} \times L_{z}\right)=(2 \pi \times 2 \pi \times 2 \pi)$ using $N_{x} \times N_{y} \times N_{z}=512 \times 512 \times 512$ collocation points. The forcing developed by Alvelius (1999) is used to sustain the TKE and the forcing energy is concentrated in wave number $k_{p}=7$. The kinematic viscosity is $\nu=0.03$. The initial HIT simulation was designed to produce initial turbulent fields with Reynolds number based on the Taylor microscale equal to $R e_{\lambda}=q \lambda_{\infty} / \nu=30$.

Some time after the HIT simulation has attained a statistically steady state with approximately constant TKE, and at a particular time (arbitrarily labelled $t=0$ ), the kinetic energy forcing is deactivated and all the velocity components are artificially set to zero $(\boldsymbol{u}(\boldsymbol{x}, t=0)=0)$ for all grid points lying outside a central region of the computational box $(z>+0.7 \pi$ and $z<-0.7 \pi)$. This corresponds to 332 collocation points, i.e. roughly $65 \%$ of the flow points in the $z$ direction.

A new phase of the simulation is then started (for $t>0$ ) departing from this initial condition, with the evolving flow having three distinct regions: $(i)$ an isotropic turbulence region, at the centre of the computational domain $(-0.7 \pi<z<+0.7 \pi)$, (ii) an irrotational region to one side of the central turbulence region $(-2 \pi<z<-0.7 \pi)$ and, (iii) another irrotational region to the other side of the central turbulence region $(+0.7 \pi<z<+2 \pi)$. (Note that the $z$ used here differs from that adopted in the RDT calculations). In this SFT simulation, the central turbulence region expands into the two irrotational regions in the absence of mean shear. Even though the boundary conditions 
along the $z$ direction are periodic, these boundaries are very far away from the central turbulent region, and no effect on the spreading rate of the central turbulence could be observed, even in very fine-scale statistics, for the duration of the simulations, which was of roughly one eddy turn-over time.

Clearly, this artificial initialization generates discontinuities in the velocity components in the two planes defined by $z= \pm 0.7 \pi$, however the pressure redistributes the energy among the velocity components, so that at the end of the first iteration the velocity field is divergence-free. Perot \& Moin (1995) carried out similar simulations, and observed that the key to eliminate the initial velocity discontinuities is drastically reducing the time step of the simulations. Several numerical tests were carried out to assess the time step needed to smooth the initial velocity discontinuities. In these tests, the vorticity field was inspected for possible discontinuities, and it was observed that by using a time step of $\Delta t=1 \times 10^{-3}$ all the initial vorticity field discontinuities were smoothed out very quickly (in roughly 3 time steps). This time step was therefore used in the simulations.

Resolution tests were also undertaken, to define the grid resolution needed for the SFT simulations. Simulations using four different resolutions were carried out, with $N_{x} \times N_{y} \times$ $N_{z}=128^{3}, 256^{3}, 384^{3}$, and $512^{3}$ collocation points. The resolution tests consisted of analyzing the conditional mean profiles (in relation to distance from the $\mathrm{T} / \mathrm{NT}$ interface) of the vorticity variance $\overline{|\boldsymbol{\omega}|^{2}}$ and viscous dissipation rate $\varepsilon$, quantities that have sharp maxima near the T/NT interface (as will be seen). Specifically, the maxima of these quantities and the asymptotic behaviour of $\varepsilon$ in the irrotational flow region were assessed. The results showed that neither the $128^{3}$ nor the $256^{3}$ simulations were able to capture the correct behaviour of these quantities, giving different values for the magnitude of their maxima relative to simulations at higher resolutions. As expected, the maxima of $\overline{|\boldsymbol{\omega}|^{2}}$ and $\varepsilon$ at $256^{3}$ resolution, and especially those at $128^{3}$ resolution, were rather lower than at $384^{3}$ or $512^{3}$ resolutions. Even though simulations at all resolutions display power laws for $\varepsilon$ in the irrotational flow region, the corresponding exponent does not agree with the theoretical one derived later in this paper for large $|z|$, except at the $512^{3}$ and $384^{4}$ resolutions. Furthermore, at both $128^{2}$ and $256^{3}$ resolutions, the same statistics display in the far-field irrotational region characteristic upturns, indicating that the simulations were unable to capture the correct asymptotic behaviour for large $|z|$. These deficiencies are absent from the simulations with $384^{3}$ and $512^{3}$ collocation points. Indeed, both of these simulations show virtually the same result, i.e. attain the same maxima in $\overline{|\boldsymbol{\omega}|^{2}}$ and $\varepsilon$, while displaying the same power law for $\varepsilon$ in the irrotational region for large values of $|z|$, which agrees very well with the theoretical prediction that will be derived later, namely $\varepsilon \sim z^{-6}$ for $z \rightarrow-\infty$. Moreover, we observe that this power law is well satisfied up to the end of the computational box in the irrotational regions, which again demonstrates that the imposition of periodic boundary conditions does not affect the fine-scale statistics. The simulation with $512^{3}$ points was therefore chosen, since grid convergence was observed from $384^{3}$ to $512^{3}$.

We note in passing that an estimation of the resolution needed to capture the thickness of the vorticity jump across the T/NT interface can be made by using the results recently published by da Silva \& Taveira (2010), where it is shown that for SFT this thickness is equal to the radius of the 'worms' from isotropic turbulence. As shown by Jiménez \& Wray (1998), that radius is $R \approx 5 \eta$ for a wide range of Reynolds numbers $\left(R e_{\lambda}=37-168\right)$. Since in the present case the Kolmogorov micro-scale is $\eta=1.996 \times 10^{-2}$ (taking the value computed for the simulation with $512^{3}$ grid points as reference), the number of points per radius is $\approx 5 \eta / \Delta x=5 \eta /\left(2 \pi / N_{x}\right)$. This gives approximately $2.0,4.1,6.1$, and 8.1 points for the simulations with $128^{3}, 256^{3}, 384^{3}$, and $512^{3}$ resolution, respectively. 


\section{Results}

Theoretical results from inviscid RDT, which are not directly comparable with DNS, will be presented in $\S 3.1$. These results yield several predictions that are independent of the form of the assumed turbulence energy spectrum, which would be masked if the dynamics of the VBL was considered. In $\S 3.2$, results from viscous RDT, including the evolution of the VBL, will be compared with DNS.

\subsection{Inviscid RDT results}

Flow statistics from inviscid RDT would correspond to a time immediately after sudden boundary insertion, when the VBL has zero thickness, i.e. $t=0^{+}$. Previous RDT studies suggest that turbulence in equilibrium at later times retains many characteristics of this virtual inviscid state, perhaps because the same boundary conditions at the interface remain valid (Hunt \& Graham 1978). In this section, attention will be focused on the velocity and strain rate covariances of the flow, as well as on the mean pressure and turbulence length scales. The asymptotic behaviour of the flow in the non-turbulent zone as $z \rightarrow-\infty$ will also be addressed.

\subsubsection{Reynolds stresses}

For isotropic turbulence, such as considered here at $z \rightarrow+\infty$ (or in irrotational flow outside this turbulence), the Reynolds stresses reduce to the velocity variances, since $\overline{u_{i} u_{j}}=0$ if $i \neq j$ by symmetry. The flow is also everywhere statistically isotropic in the $x-y$ plane, therefore $\overline{u_{ \pm}^{2}}=\overline{v_{ \pm}^{2}}$. The tangential velocity variance takes the form

$$
\begin{aligned}
\overline{u_{-}^{2}}= & \frac{1}{16} \int_{0}^{\pi} \int_{0}^{+\infty} E(k) \sin ^{3} \theta \mathrm{e}^{2 k \sin \theta z} \mathrm{~d} k \mathrm{~d} \theta, \quad z<0, \\
\overline{u_{+}^{2}}= & \frac{1}{4} \int_{0}^{\pi} \int_{0}^{+\infty} E(k) \sin \theta\left[\sin ^{2} \theta+2 \cos ^{2} \theta+\frac{1}{4} \sin ^{2} \theta \mathrm{e}^{-2 k \sin \theta z}\right. \\
& \left.-\sin \theta \cos \theta \mathrm{e}^{-k \sin \theta z} \sin (k \cos \theta z)\right] \mathrm{d} k \mathrm{~d} \theta, \quad z>0,
\end{aligned}
$$

while the normal velocity variance can be expressed as:

$$
\begin{aligned}
& \overline{w_{-}^{2}}=\frac{1}{8} \int_{0}^{\pi} \int_{0}^{+\infty} E(k) \sin ^{3} \theta \mathrm{e}^{2 k \sin \theta z} \mathrm{~d} k \mathrm{~d} \theta, \quad z<0, \\
& \overline{w_{+}^{2}}=\frac{1}{2} \int_{0}^{\pi} \int_{0}^{+\infty} E(k) \sin ^{3} \theta\left[1+\frac{1}{4} \mathrm{e}^{-2 k \sin \theta z}-\mathrm{e}^{-k \sin \theta z} \cos (k \cos \theta z)\right] \mathrm{d} k \mathrm{~d} \theta, \quad z>0 .
\end{aligned}
$$

The above equations were obtained from (2.23), using (2.25) and the expressions for the matrices $M_{i j}^{ \pm}$presented in the Appendix. Spherical polar coordinates were adopted to express the wavenumber in the originally $3 \mathrm{D}$ integrals, and integration over the azimuthal angle was performed analytically. The remaining integrations must, in general, be carried out numerically.

Figure 2(a) presents profiles of $\overline{u^{2}}$ and $\overline{w^{2}}$ normalized by the RMS velocity in the HIT, q. Similar results were first obtained by Carruthers \& Hunt (1986) and are cited, for example, by Bisset, Hunt \& Rogers (2002) and Hunt, Eames \& Westerweel (2006) in the context of T/NT interfaces. Obviously, as $z \rightarrow+\infty, \overline{u^{2}}, \overline{w^{2}} \rightarrow q$, and it can be shown also from (3.1)-(3.2) that

$$
\overline{u_{-}^{2}}(z=0)=\frac{1}{8} q^{2}, \quad \overline{u_{+}^{2}}(z=0)=\frac{9}{8} q^{2},
$$



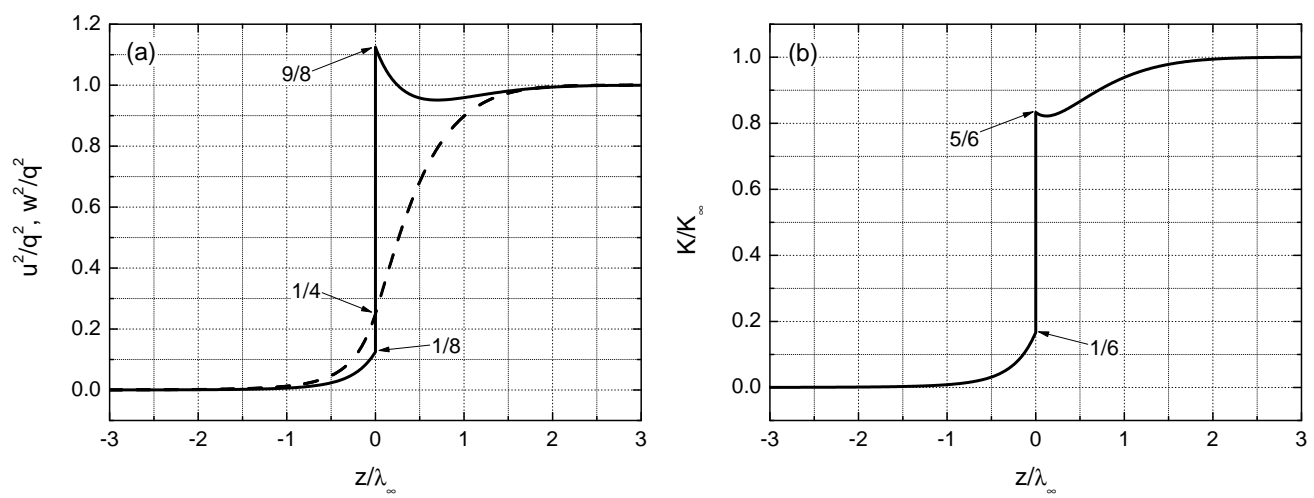

Figure 2. (a) Tangential and normal velocity variances normalized by $q$. Solid line: $\overline{u^{2}} / q^{2}$, dashed line: $\overline{w^{2}} / q^{2}$. (b) TKE normalized by its value at $z \rightarrow+\infty$. In $(a)$ and $(b)$ the values of each quantity at $z=0^{+}$and $z=0^{-}$are displayed.

$$
\overline{w_{-}^{2}}(z=0)=\overline{w_{+}^{2}}(z=0)=\frac{1}{4} q^{2} .
$$

This is a consequence of the general property that one-point turbulence statistics at $z=0$ may always be expressed in terms of their values at $z \rightarrow+\infty$, with no need to specify the form of the energy spectrum (cf. Batchelor \& Proudman 1954). These statistics only depend on the spectral form of $E(k)$ in the intermediate regions $-\infty<z<0$ or $0<z<+\infty$. This property, which also holds for the covariances of the strain rates and related quantities, gives some generality to the present calculations. It is also worth noting from (3.1) and (3.2) that, at $z<0$, i.e. in the non-turbulent zone, $\overline{u^{2}}=1 / 2 \overline{w^{2}}$. This well-known result was first derived by Phillips (1955), and is clearly consistent with figure 5 of Tordella, Iovieno \& Bailey (2008), showing the flow anisotropy at the boundary between two isotropic turbulence regions, for the case where turbulence at one side of the boundary is much weaker than at the other side.

Figure $2(b)$ presents a profile of the TKE, defined as $K=(1 / 2)\left(\overline{u^{2}}+\overline{v^{2}}+\overline{w^{2}}\right)=$ $(1 / 2)\left(2 \overline{u^{2}}+\overline{w^{2}}\right)$, where the second equality results from statistical isotropy in the $x-y$ plane. This quantity is normalized by its far-field value in the turbulent zone, namely $K_{\infty}=(3 / 2) q^{2}$. The values that $K / K_{\infty}$ takes at $z=0^{-}$and $z=0^{+}$obviously result from (3.3) and are given by

$$
K\left(z=0^{-}\right)=\frac{1}{6} K_{\infty}, \quad K\left(z=0^{+}\right)=\frac{5}{6} K_{\infty} .
$$

In fact, it is not strictly accurate to call $K$ the TKE, since this kinetic energy is not directly associated with turbulence at $z<0$, but that standard terminology will be kept here for definiteness. The asymptotic behaviour of $K$ in the non-turbulent region will be analyzed in $\S 3.1 .4$.

\subsubsection{Strain-rate covariances and dissipation rate}

Carruthers \& Hunt (1986) did not calculate the variances and covariances of turbulent strain rates, which are important for determining quantities such as the viscous dissipation rate or the Taylor microscale. In some RDT studies, this is avoided presumably because the corresponding integrals diverge for the usually employed Von Kármán energy spectrum (cf. Hunt \& Graham 1978; Fernando \& Hunt 1997). This spectrum, unlike (2.26), possesses an inertial sub-range that extends to $k \rightarrow \infty$. However, for statistics that can be calculated analytically, this problem can be precluded if they are normalized 
by their value in the bulk of the turbulence, because then the spectral dependence cancels out.

In HIT, such as is assumed to exist at $z \rightarrow+\infty$,

$$
\begin{aligned}
\overline{\left(\frac{\partial u_{i+}}{\partial x_{j}}\right)_{\infty}^{2}} & =\frac{1}{15} \frac{\varepsilon_{\infty}}{\nu} \quad \text { if } \quad i=j, \quad \overline{\left(\frac{\partial u_{i+}}{\partial x_{j}}\right)_{\infty}^{2}}=\frac{2}{15} \frac{\varepsilon_{\infty}}{\nu} \quad \text { if } \quad i \neq j, \\
\overline{\left(\frac{\partial u_{i+}}{\partial x_{j}} \frac{\partial u_{j+}}{\partial x_{i}}\right)_{\infty}} & =-\frac{1}{30} \frac{\varepsilon_{\infty}}{\nu} \quad \text { if } i \neq j,
\end{aligned}
$$

where $\varepsilon_{\infty}=\varepsilon(z \rightarrow+\infty)$, and the index-repetition convention is not used. The values of the strain rate covariances near the $\mathrm{T} / \mathrm{NT}$ interface can be expressed in terms of the same quantities. Using (2.24) and (2.25), as well as the definition for $M_{i j}^{ \pm}$(provided in the Appendix), expressions in a similar form to (3.1) and (3.2) may be obtained. These expressions are omitted here for brevity. Attention is focused in particular on $\overline{(\partial u / \partial x)^{2}}$, $\overline{(\partial u / \partial y)^{2}}, \overline{(\partial u / \partial z)^{2}}, \overline{(\partial w / \partial x)^{2}}, \overline{(\partial w / \partial z)^{2}}, \overline{(\partial u / \partial y)(\partial v / \partial x)}$ and $\overline{(\partial u / \partial z)(\partial w / \partial x)}$, since other strain variances and covariances are related to these ones by symmetry. From the resulting expressions, it can be shown that

$$
\begin{aligned}
& \overline{\left(\frac{\partial u_{-}}{\partial x}\right)^{2}}(z=0)=\frac{3}{120} \frac{\varepsilon_{\infty}}{\nu}, \quad \overline{\left(\frac{\partial u_{-}}{\partial y}\right)^{2}}(z=0)=\frac{1}{120} \frac{\varepsilon_{\infty}}{\nu} \\
& \overline{\left(\frac{\partial u_{-}}{\partial z}\right)^{2}}(z=0)=\frac{1}{30} \frac{\varepsilon_{\infty}}{\nu}, \quad \overline{\left(\frac{\partial w_{-}}{\partial x}\right)^{2}}(z=0)=\frac{1}{30} \frac{\varepsilon_{\infty}}{\nu}, \quad \overline{\left(\frac{\partial w_{-}}{\partial z}\right)^{2}}(z=0)=\frac{1}{15} \frac{\varepsilon_{\infty}}{\nu} \\
& \frac{\partial u_{-}}{\partial y} \frac{\partial v_{-}}{\partial x}(z=0)=\frac{1}{120} \frac{\varepsilon_{\infty}}{\nu}, \quad \overline{\frac{\partial u_{-}}{\partial z} \frac{\partial w_{-}}{\partial x}}(z=0)=\frac{1}{30} \frac{\varepsilon_{\infty}}{\nu} \\
& \frac{\left(\frac{\partial u_{+}}{\partial x}\right)^{2}}{(z=0)}=\frac{11}{120} \frac{\varepsilon_{\infty}}{\nu}, \quad \overline{\left(\frac{\partial u_{+}}{\partial y}\right)^{2}}(z=0)=\frac{17}{120} \frac{\varepsilon_{\infty}}{\nu}, \quad \overline{\left.\frac{\partial u_{+}}{\partial z}\right)^{2}}(z=0)=\frac{3}{15} \frac{\varepsilon_{\infty}}{\nu}, \quad \overline{\left(\frac{\partial w_{+}}{\partial x}\right)^{2}}(z=0)=\frac{1}{30} \frac{\varepsilon_{\infty}}{\nu}, \quad \overline{\left(\frac{\partial w_{+}}{\partial z}\right)^{2}}(z=0)=\frac{2}{15} \frac{\varepsilon_{\infty}}{\nu} \\
& \frac{\partial u_{+}}{\partial y} \frac{\partial v_{+}}{\partial x}(z=0)=-\frac{1}{40} \frac{\varepsilon_{\infty}}{\nu}, \quad \frac{\partial u_{+}}{\partial z} \frac{\partial w_{+}}{\partial x}(z=0)=-\frac{1}{20} \frac{\varepsilon_{\infty}}{\nu} .
\end{aligned}
$$

In (3.6), $\overline{\left(\partial u_{-} / \partial y\right)\left(\partial v_{-} / \partial x\right)}=\overline{\left(\partial u_{-} / \partial y\right)^{2}}$ and $\left.\overline{\left(\partial u_{-} / \partial z\right)^{2}}=\overline{\left(\partial w_{-} / \partial x\right.}\right)^{2}=\overline{\left(\partial u_{-} / \partial z\right)\left(\partial w_{-} / \partial x\right)}$, because $\partial v / \partial x=\partial u / \partial y$ and $\partial u / \partial z=\partial w / \partial x$ in irrotational flow. Of course, this property extends to all $z<0$.

Figure $3(a-g)$ shows profiles of the independent variances and covariances of the strain rates, normalized by their modulus at $z \rightarrow+\infty$. Their values at $z=0^{+}$and $z=0^{-}$ result directly from (3.6)-(3.7).

The viscous dissipation rate of TKE is defined as

$$
\varepsilon=2 \nu \overline{s_{i j} s_{i j}}, \quad \text { where } \quad s_{i j}=\frac{1}{2}\left(\frac{\partial u_{i}}{\partial x_{j}}+\frac{\partial u_{j}}{\partial x_{i}}\right), \quad \text { or } \quad \varepsilon=\nu\left[\overline{\left(\frac{\partial u_{i}}{\partial x_{j}}\right)^{2}}+\overline{\frac{\partial u_{i}}{\partial x_{j}} \frac{\partial u_{j}}{\partial x_{i}}}\right]
$$

(where the index-repetition convention is used) or, by symmetry, as

$$
\varepsilon=\nu\left[4 \overline{\left(\frac{\partial u}{\partial x}\right)^{2}}+2 \overline{\left(\frac{\partial u}{\partial y}\right)^{2}}+2 \overline{\left(\frac{\partial u}{\partial z}\right)^{2}}+2 \overline{\left(\frac{\partial w}{\partial x}\right)^{2}}+2 \overline{\left(\frac{\partial w}{\partial z}\right)^{2}}+2 \overline{\frac{\partial u}{\partial y} \frac{\partial v}{\partial x}}+4 \overline{\frac{\partial u}{\partial z} \frac{\partial w}{\partial x}}\right] .
$$



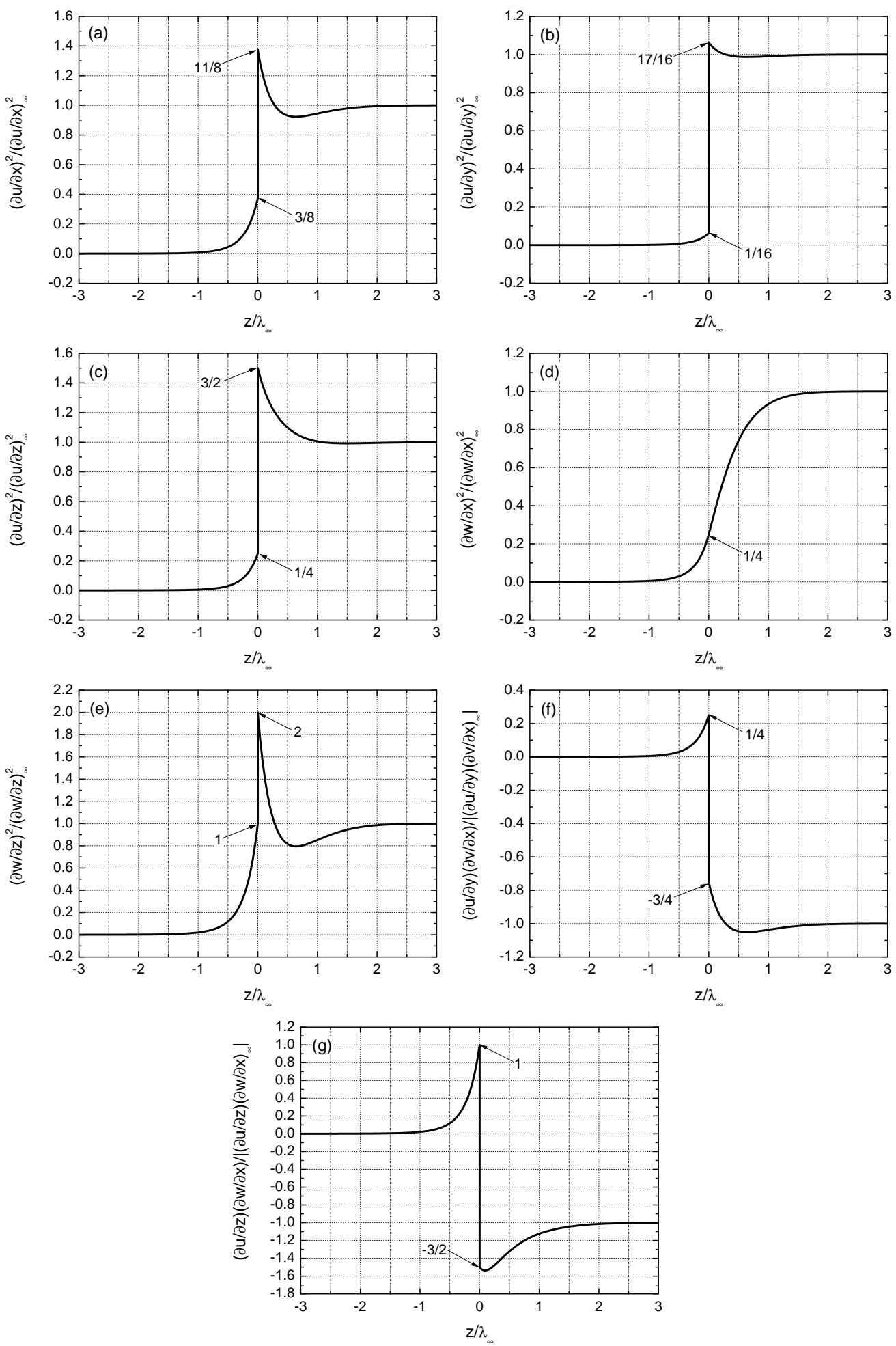

FIGURE 3. Variances and covariances of the strain rates normalized by their absolute values at $z \rightarrow+\infty$. The values of each quantity at $z=0^{+}$and $z=0^{-}$are displayed. $(a) \overline{(\partial u / \partial x)^{2}},(b)$ $\overline{(\partial u / \partial y)^{2}},(c) \overline{(\partial u / \partial z)^{2}},(d) \overline{(\partial w / \partial x)^{2}},(e) \overline{(\partial w / \partial z)^{2}},(f) \overline{(\partial u / \partial y)(\partial v / \partial x)},(g) \overline{(\partial u / \partial z)(\partial w / \partial x)}$. 


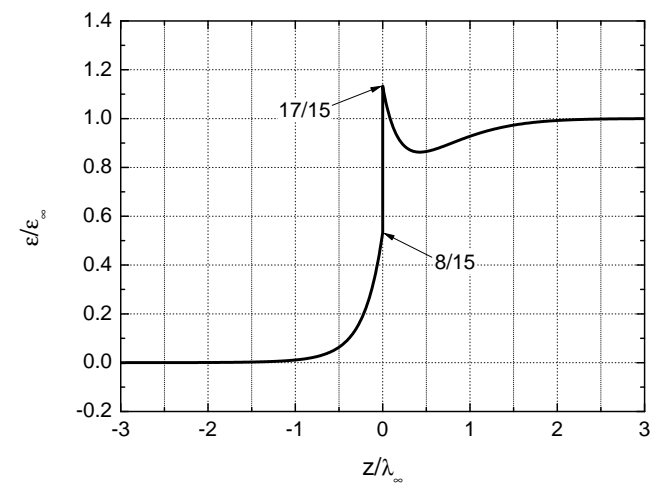

Figure 4 . Dissipation rate $\varepsilon$ normalized by its value at $z \rightarrow+\infty$. The values of the dissipation rate at $z=0^{+}$and $z=0^{-}$are displayed.

This quantity, which may be calculated using the variances and covariances of the strain rates presented in figure $3(a-g)$, is shown in figure 4. Again, the values of $\varepsilon$ at $z=0^{+}$or $z=0^{-}$result from the corresponding limits in (3.6)-(3.7), and are:

$$
\varepsilon\left(z=0^{+}\right)=\frac{17}{15} \varepsilon_{\infty}, \quad \varepsilon\left(z=0^{-}\right)=\frac{8}{15} \varepsilon_{\infty} .
$$

The dissipation rate has a sharp peak at $z=0^{+}$, and a relative minimum at $z / \lambda_{\infty} \approx 0.4$. In the non-turbulent zone, $\varepsilon$ is not zero, but rather gradually decays to zero as $z \rightarrow-\infty$. This asymptotic behaviour will be analyzed in $\S 3.1 .4$. It is worth mentioning that (3.8) is the actual dissipation rate and not the pseudo-dissipation rate, investigated by Perot \& Moin (1995) or Teixeira \& Belcher (2000).

In turbulence studies, the relation $\varepsilon=\nu \overline{|\boldsymbol{\omega}|^{2}}$ is often invoked. For example, in Hunt (1984) and Carruthers \& Hunt (1986), the observed constancy of $\varepsilon$ in turbulent regions is used to justify the adoption of a constant turbulent vorticity level. It must be noted however, that this relation is only strictly valid for HIT. The turbulent vorticity variance is defined as

$$
\overline{|\boldsymbol{\omega}|^{2}}=\overline{\left(\frac{\partial u_{i}}{\partial x_{j}}\right)^{2}}-\overline{\frac{\partial u_{i}}{\partial x_{j}} \frac{\partial u_{j}}{\partial x_{i}}}=2 \overline{\left(\frac{\partial u}{\partial y}\right)^{2}}+2 \overline{\left(\frac{\partial u}{\partial z}\right)^{2}}+2 \overline{\left(\frac{\partial w}{\partial x}\right)^{2}}-2 \overline{\frac{\partial u}{\partial y} \frac{\partial v}{\partial x}}-4 \overline{\frac{\partial u}{\partial z} \frac{\partial w}{\partial x}} .
$$

In the presence of inhomogeneities, such as introduced by a boundary, the correct relation between $\varepsilon$ and $\overline{|\boldsymbol{\omega}|^{2}}$ is

$$
\varepsilon=\nu\left[\overline{|\boldsymbol{\omega}|^{2}}+2 \frac{\partial^{2}}{\partial x_{i} \partial x_{j}}\left(\overline{u_{i} u_{j}}\right)\right]=\nu\left(\overline{|\boldsymbol{\omega}|^{2}}+2 \frac{\partial^{2} \overline{w^{2}}}{\partial z^{2}}\right),
$$

where the second equality is valid in the case of a flow that is statistically homogeneous in the $x-y$ plane. From (3.12), it follows that, in the irrotational flow zone of the present model, all dissipation is due to a term whose form is similar to a diffusion. Da Silva \& Reis (2011) argue that the 'irrotational' dissipation observed near the T/NT interface is induced by the presence of large vorticity structures in that region.

\subsubsection{Mean pressure}

Although the present model neglects any mean flow, hints as to what entrainment velocity might be generated by the perturbed flow can be inferred from the mean pressure 


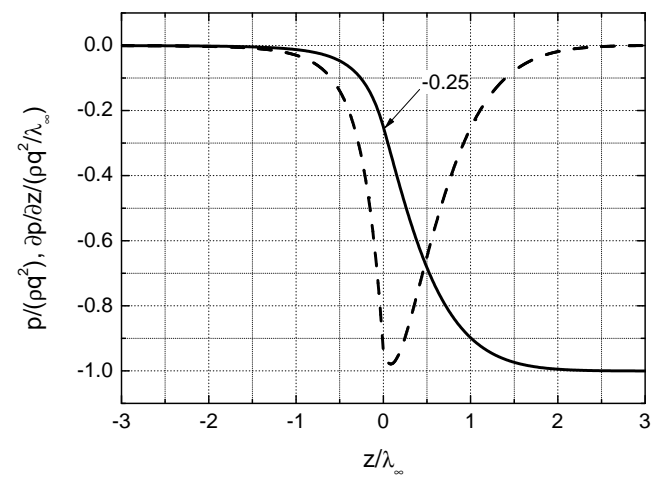

Figure 5 . Mean pressure normalized by its absolute value at $z \rightarrow+\infty$ (solid line), and the corresponding pressure gradient (dashed line). The value of $\bar{p}$ at $z=0$ is displayed.

distribution. The pressure can be related to the velocity field through

$$
\nabla^{2} p=-\rho \frac{\partial u_{i}}{\partial x_{j}} \frac{\partial u_{j}}{\partial x_{i}}
$$

which was obtained by taking the divergence of (2.2) and using (2.3). Taking the ensemble average of (3.13), noting that, in the present model, mean quantities only depend on $z$, and using again the symmetries resulting from isotropy in the $x-y$ plane, (3.13) becomes:

$$
\frac{\partial^{2} \bar{p}}{\partial z^{2}}=-\rho\left[2 \overline{\left(\frac{\partial u}{\partial x}\right)^{2}}+\overline{\left(\frac{\partial w}{\partial z}\right)^{2}}+2 \overline{\frac{\partial u}{\partial y} \frac{\partial v}{\partial x}}+4 \overline{\frac{\partial u}{\partial z} \frac{\partial w}{\partial x}}\right] .
$$

This equation must be solved separately for $\bar{p}_{+}$in the region $z>0$ and for $\bar{p}_{-}$in the region $z<0$. The boundary conditions are that both $\bar{p}$ and $\partial \bar{p} / \partial z$ be continuous at $z=0$, and tend to zero as $z \rightarrow-\infty$ (a constant or hydrostatic component is implicitly subtracted from this mean pressure). The final result is:

$$
\begin{aligned}
\bar{p}_{-}= & -\frac{1}{8} \rho \int_{0}^{\pi} \int_{0}^{+\infty} E(k) \sin ^{3} \theta \mathrm{e}^{2 k \sin \theta z} \mathrm{~d} k \mathrm{~d} \theta, \\
\bar{p}_{+}= & -\frac{1}{4} \rho q^{2}+\frac{1}{8} \rho \int_{0}^{\pi} \int_{0}^{+\infty} E(k) \sin ^{3} \theta\left\{1-\mathrm{e}^{-2 k \sin \theta z}\right. \\
& \left.-4\left[1-\mathrm{e}^{-k \sin \theta z} \cos (k \cos \theta z)\right]\right\} \mathrm{d} k \mathrm{~d} \theta .
\end{aligned}
$$

From (3.15), it can be shown that

$$
\bar{p}(z=0)=-\frac{1}{4} \rho q^{2}, \quad \bar{p}_{+}(z \rightarrow+\infty)=-\rho q^{2} .
$$

For all $z<0$, it can also be shown that

$$
\bar{p}_{-}=-2 \rho \overline{u_{-}^{2}}=-\rho K,
$$

which is just an averaged version of Bernoulli's equation for irrotational flow. Tordella et al. (2008) found equivalent relations by analyzing the momentum balance in a direction perpendicular to the boundary separating two regions of isotropic turbulence with different intensities, in the absence of mean entrainment. Note that, by (3.16), for $z \rightarrow+\infty$, $\bar{p}_{+}=-(2 / 3) \rho K$, i.e. Bernoulli's equation is not satisfied in the turbulent region because the flow is not irrotational.

Figure 5 presents profiles of $\bar{p}$ and $\partial \bar{p} / \partial z$. It can be seen that the normal pressure 
gradient attains a maximum of its absolute value slightly inside the turbulent zone. The fact that, in figure 5, the mean pressure becomes negative inside the turbulent zone appears to indicate that a mean flow into the turbulence (i.e. an entrainment velocity) should be induced if the the flow was free to evolve from an initially quiescent mean state. However, the situation may be more complex, as shown by Tordella et al. (2008).

\subsubsection{Asymptotic behaviour of turbulence statistics in the irrotational region}

Phillips (1955) was the first to study theoretically the asymptotic behaviour of velocity fluctuations outside a turbulent zone, concluding that the velocity variances decay as $z^{-4}$ for sufficiently large distances. This power law decay results directly from the assumed lower wavenumber limit of the energy spectrum $\left(\propto k^{4}\right)$. In the present model, that result will be reproduced, as follows. If the integral for $\overline{u_{-}^{2}}$ is not expressed in spherical polar coordinates, as in (3.1), but rather in cylindrical coordinates, then

$$
\overline{u_{-}^{2}}=\frac{1}{16 \pi} \int_{0}^{2 \pi} \int_{0}^{+\infty} \int_{-\infty}^{+\infty} \frac{E(k)}{k^{4}} k_{12}^{3} \cos ^{2} \alpha \mathrm{e}^{2 k_{12} z} \mathrm{~d} k_{3} \mathrm{~d} k_{12} \mathrm{~d} \alpha,
$$

where $k_{1}=k_{12} \cos \alpha$ and $k_{2}=k_{12} \sin \alpha$. Using (2.26), the integrations over $k_{3}$ and $\alpha$ may be performed analytically, yielding

$$
\overline{u_{-}^{2}}=\frac{q^{2}}{16} \int_{0}^{+\infty} k_{12}^{\prime 3} \mathrm{e}^{2 k_{12}^{\prime} z^{\prime}-\frac{1}{2} k_{12}^{\prime 2}} \mathrm{~d} k_{12}^{\prime},
$$

where $k_{12}^{\prime}=k_{12} \lambda_{\infty}$ and $z^{\prime}=z / \lambda_{\infty}$. For $\left|z^{\prime}\right| \gg 1$, the integral in (3.19) receives dominant contributions from relatively low values of $k_{12}^{\prime}$, for which $\exp \left(-\frac{1}{2} k_{12}^{\prime 2}\right) \approx 1$. In that limit, (3.19) may be integrated to yield

$$
\overline{u_{-}^{2}} \sim \frac{3 q^{2}}{128 z^{\prime 4}}
$$

as expected. Since $\overline{w_{-}^{2}}=2 \overline{u_{-}^{2}},(3.20)$ leads to

$$
\overline{w_{-}^{2}} \sim \frac{3 q^{2}}{64 z^{\prime 4}}, \quad \text { and } \quad K \sim \frac{3 q^{2}}{64 z^{\prime 4}}, \quad \text { or } \quad K \sim \frac{1}{32 z^{\prime 4}} K_{\infty} .
$$

Unlike the values of the normalized variances at $z=0^{+}$or $z=0^{-}$, the proportionality constants in (3.20)-(3.21) depend on the particular form chosen for $E(k)$.

Figure $6(a)$ shows $K$ normalized by $K_{\infty}$ in the non-turbulent flow zone. The asymptotic behaviour in the two limits $z=0$ and $z \rightarrow-\infty$ is also indicated.

Using (3.12) and (3.21), the asymptotic behaviour of the viscous dissipation rate as $z \rightarrow-\infty$ may be readily obtained as

$$
\varepsilon \sim \frac{15}{8} \frac{q^{2}}{\lambda_{\infty}^{2}} \frac{1}{z^{\prime 6}}, \quad \text { or } \quad \varepsilon=\frac{1}{8 z^{\prime 6}} \varepsilon_{\infty},
$$

where $\varepsilon_{\infty}=15 q^{2} / \lambda_{\infty}^{2}$. Figure $6(b)$ shows $\varepsilon$ normalized by $\varepsilon_{\infty}$ in the non-turbulent flow zone.

Finally, figure $6(c)$ shows a profile of the absolute value of the mean pressure in the same region of the flow. The mean pressure may be obtained directly from (3.17), yielding, in the limit $z \rightarrow-\infty$

$$
\bar{p} \sim-\frac{3}{64 z^{\prime 4}} \rho q^{2} .
$$

It therefore has a similar asymptotic dependence as the velocity variances or $K$, which is expectable from Bernoulli's equation. 

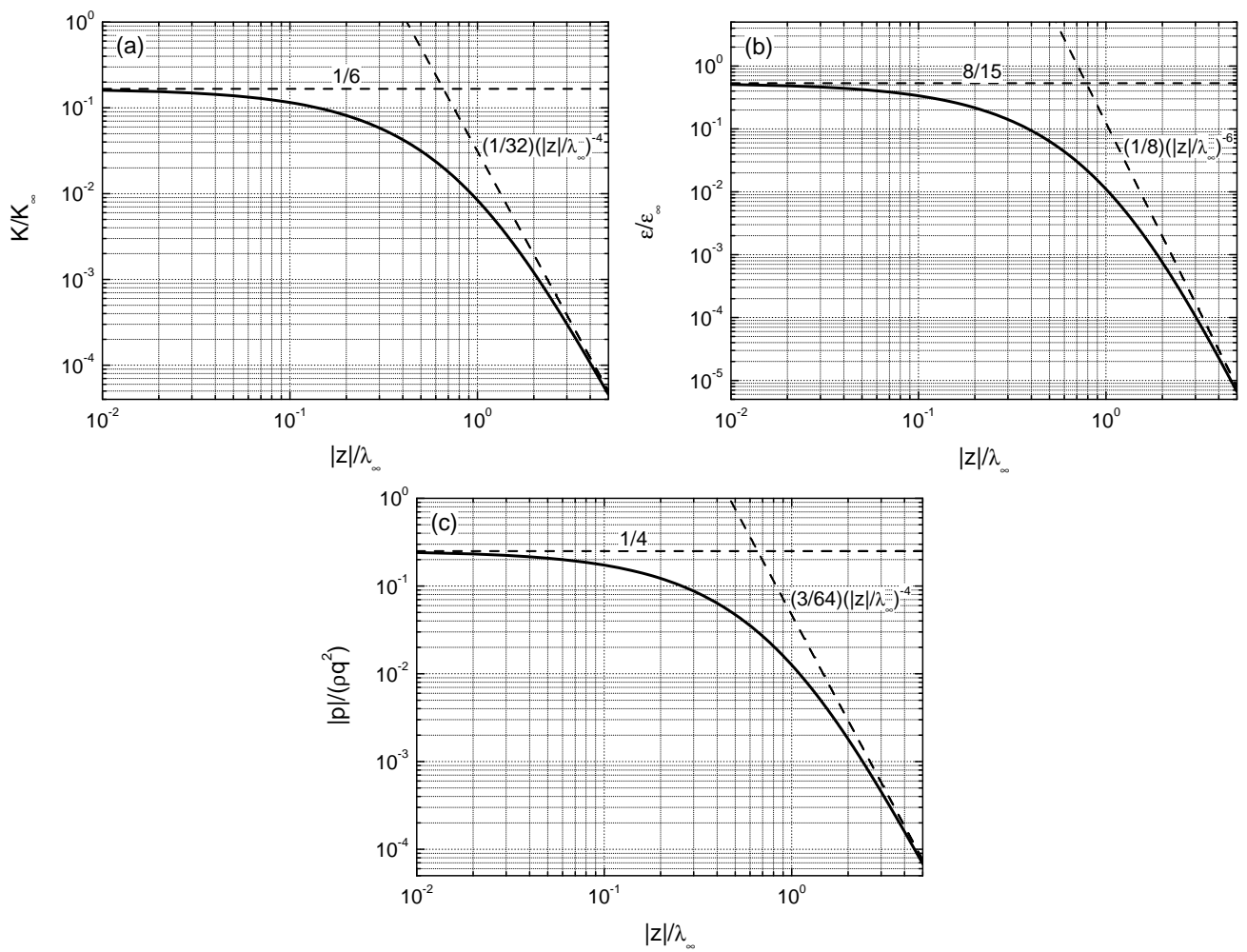

Figure 6 . Statistics at $z<0$ normalized by their values at $z \rightarrow+\infty$. (a) TKE, (b) dissipation rate $\varepsilon,(c)$ absolute mean pressure $|\bar{p}|$. The values of each quantity at $z=0$ and corresponding asymptotes for $z \rightarrow-\infty$ are displayed.

\subsubsection{Turbulence length scales}

Finally, various flow length scales will be analyzed. Integral length scales, for example, are defined along a particular spatial direction. Since in the present model the flow is statistically homogeneous and isotropic in the $x-y$ plane, the chosen direction may be $x$, without any loss of generality. The integral length scales are then given by

$$
L_{i i}^{x}=\frac{\int_{0}^{+\infty} \overline{u_{i}(x, y, z) u_{i}(x+r, y, z)} \mathrm{d} r}{\overline{u_{i}^{2}}}=\pi \frac{\iint\left(M_{i k}^{*} M_{i l} \Phi_{k l}^{(H)}\right)\left(k_{1}=0\right) \mathrm{d} k_{2} \mathrm{~d} k_{3}}{\overline{u_{i}^{2}}},
$$

where $i=1,2,3$, respectively, for the $u, v$ and $w$ velocity fluctuations.

Figure $7(a-c)$ shows profiles of these integral length scales normalized by their value at $z \rightarrow+\infty$. The behaviour of the length scales in the turbulent zone is unremarkable, though it can be shown that

$$
\begin{aligned}
& L_{11}^{x}\left(z=0^{+}\right)=\frac{8}{9} L_{11 \infty}^{x}, \quad L_{22}^{x}\left(z=0^{-}\right)=2 L_{22 \infty} \\
& L_{22}^{x}\left(z=0^{+}\right)=\frac{10}{9} L_{22 \infty}^{x}, \quad L_{33}^{x}(z=0)=L_{33 \infty}^{x} .
\end{aligned}
$$

In the non-turbulent zone, however. $L_{11}^{x}$ is zero and both $L_{22}^{x}$ and $L_{33}^{x}$ asymptotically grow linearly as $z \rightarrow-\infty$. The first aspect is due to the fact that the integral of the covariance in the numerator of (3.24) is always zero for irrotational flow. The asymptotic 

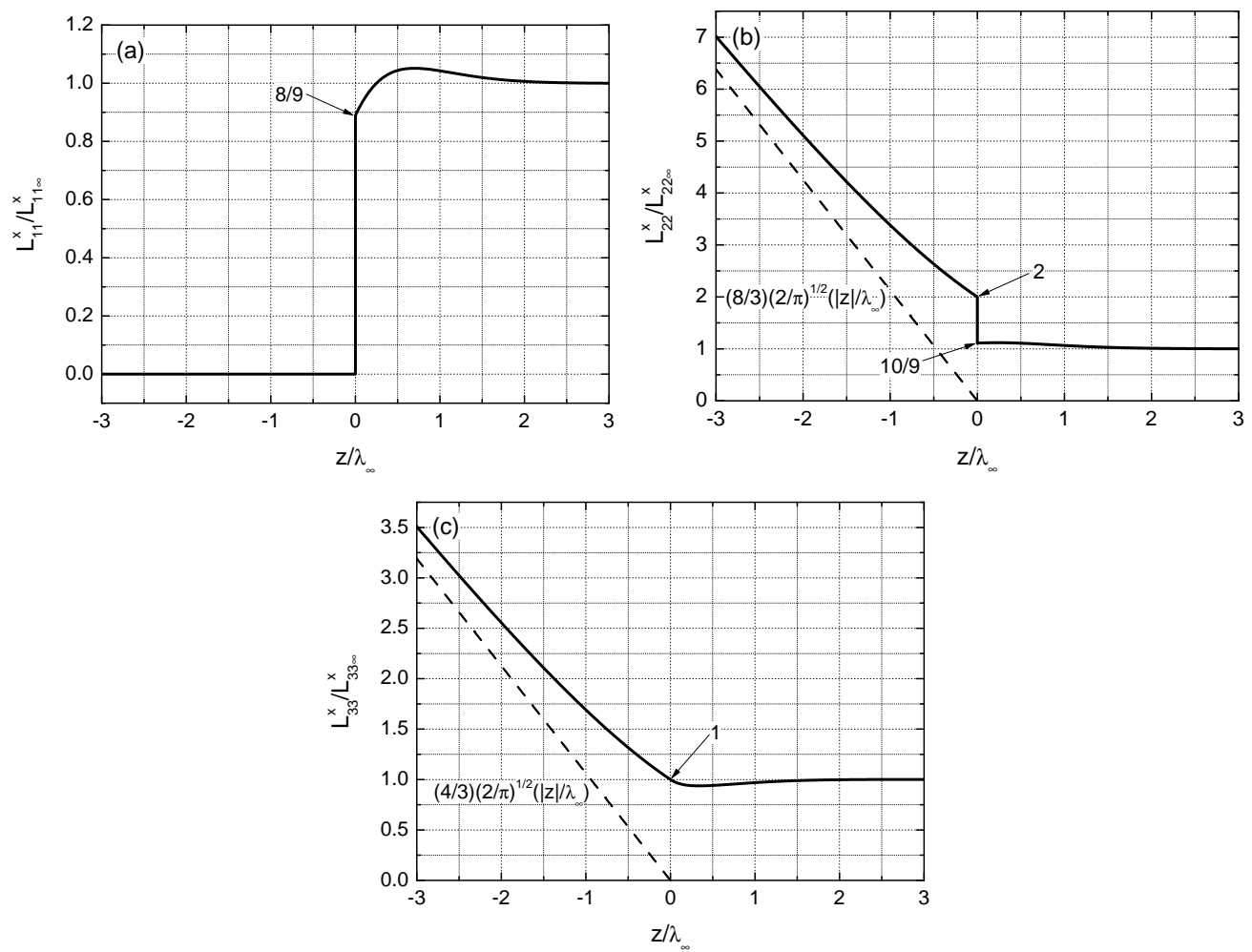

FiguRE 7 . Integral length scales normalized by their values at $z \rightarrow+\infty$. (a) $L_{11}^{x}$, (b) $L_{22}^{x},(c)$ $L_{33}^{x}$. The values of each quantity at $z=0^{+}$and $z=0^{-}$, as well as the corresponding asymptotes as $z \rightarrow-\infty$, are displayed.

behaviour of $L_{22}^{x}$ and $L_{33}^{x}$ can be shown to be

$$
L_{22}^{x} \sim \frac{8}{3}\left(\frac{2}{\pi}\right)^{1 / 2}\left|z^{\prime}\right| L_{22 \infty}^{x}, \quad L_{33}^{x} \sim \frac{4}{3}\left(\frac{2}{\pi}\right)^{1 / 2}\left|z^{\prime}\right| L_{33 \infty}^{x}=\frac{1}{2} L_{22}^{x}
$$

Physically, this behaviour is a consequence of the fact that in the non-turbulent flow zone the scale of the velocity fluctuations that manage to reach a certain distance from the $\mathrm{T} / \mathrm{NT}$ interface is proportional to that distance

Another important length scale is the Taylor microscale:

$$
\lambda=\left[\frac{\overline{u^{2}}}{\overline{\left(\frac{\partial u}{\partial x}\right)^{2}}}\right]^{1 / 2},
$$

which is defined here according to Tennekes \& Lumley (1972). Since the $x$ and $y$ directions are the only statistically homogeneous ones and the flow is isotropic in the $x-y$ plane, (3.27) is as general as possible.

From (3.3) and (3.6)-(3.7), it is possible to deduce that

$$
\lambda_{+}(z=0)=\left(\frac{9}{11}\right)^{1 / 2} \lambda_{\infty}, \quad \lambda_{-}(z=0)=\left(\frac{1}{3}\right)^{1 / 2} \lambda_{\infty}
$$

where the subscripts - and + refer to the regions $z<0$ and $z>0$, respectively. In the 

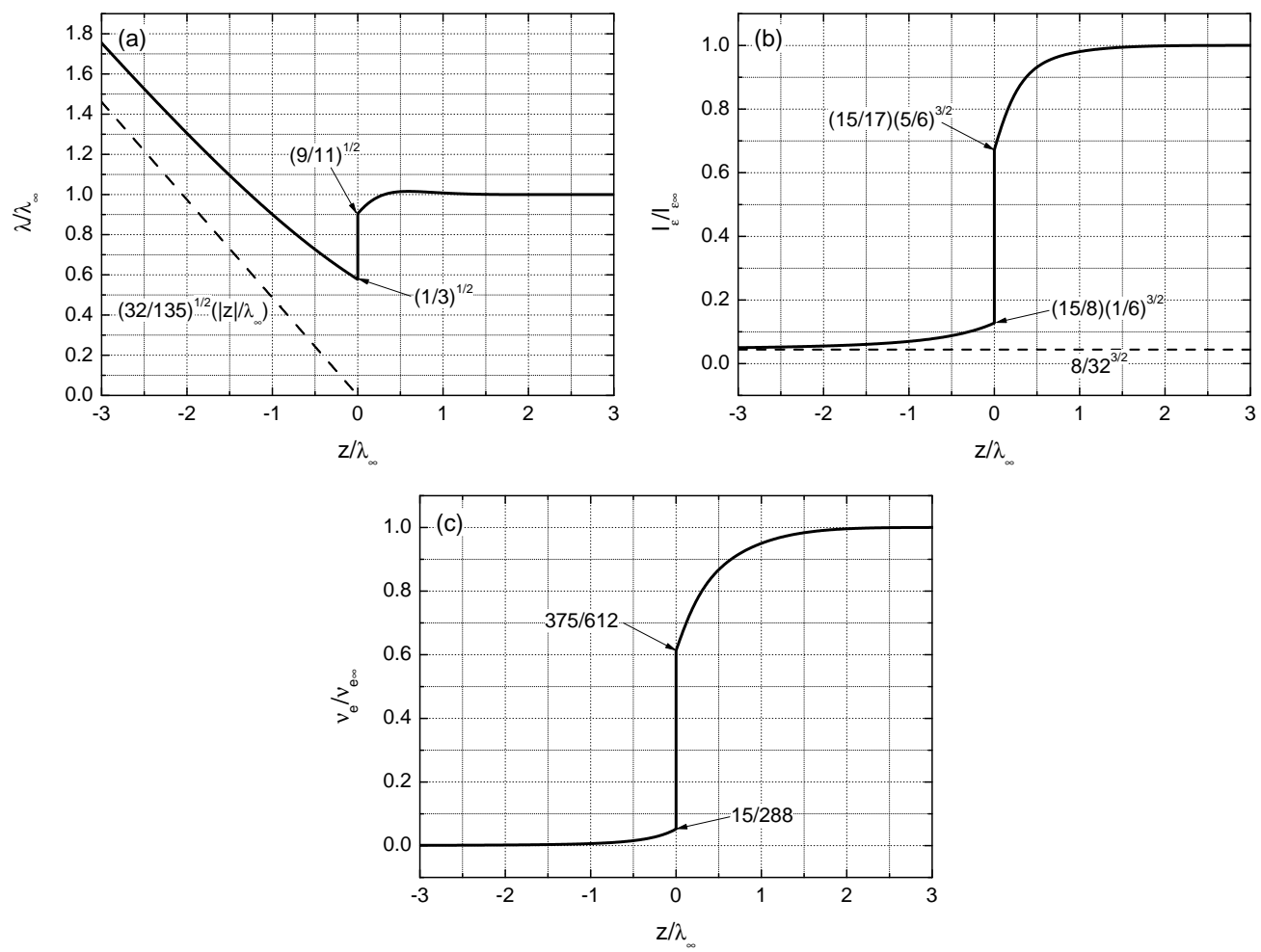

Figure 8. (a) Taylor microscale, (b) dissipation length scale, and (c) eddy viscosity, normalized by their values at $z \rightarrow+\infty$. The values of each quantity at $z=0^{+}$and $z=0^{-}$, and the corresponding asymptotes as $z \rightarrow-\infty$ are displayed.

asymptotic limit $z \rightarrow-\infty$, on the other hand,

$$
\lambda_{-} \sim\left(\frac{32}{135}\right)^{1 / 2}\left|z^{\prime}\right| \lambda_{\infty} .
$$

So the Taylor microscale behaves qualitatively like $L_{22}^{x}$ and $L_{33}^{x}$ in this limit, growing linearly with distance from the $\mathrm{T} / \mathrm{NT}$ interface. A profile of the Taylor microscale is shown in figure $8(a)$.

Two quantities mentioned by Bisset et al. (2002), which are of relevance in numerical modelling of turbulent flows, are the dissipation length scale, $l_{\varepsilon}=K^{3 / 2} / \varepsilon$, and the eddy viscosity, $\nu_{e}=K^{2} / \varepsilon$. These two quantities, whose values at $z=0^{+}$or $z=0^{-}$and asymptotic behaviours for $z \rightarrow-\infty$ can be deduced from those of $K$ and $\varepsilon$ (see (3.3), (3.6)-(3.7), (3.21) and (3.22)) are presented in figure $8(b-c)$. While in the present model $\nu_{e}$ decays to zero proportionally to $z^{-2}$ as $z \rightarrow-\infty$, the dissipation length scale attains the non-zero value $l_{\varepsilon}=8 / 32^{\frac{3}{2}} l_{\varepsilon \infty}$ in this limit (where $l_{\varepsilon \infty}$ is the corresponding value at $z \rightarrow+\infty)$. Therefore, its behaviour is qualitatively different from that of either $L_{11}^{x}, L_{22}^{x}$, $L_{33}^{x}$ or $\lambda$.

\subsection{Viscous results}

Results from the full RDT model including VBL dynamics are now compared with DNS data. The theoretical expressions for the various statistics presented in $\S 3.1$ are rather lengthy in the viscous case and will not be reproduced here. They can be obtained straightforwardly from (2.23) and (2.24), using (2.25), (2.26) and the expressions for $M_{i j}^{ \pm}$ 
provided in the Appendix. The corresponding integrals may also be expressed in spherical polar coordinates, and calculated analytically for the azimuthal angle.

As in $\S 3.1$, distances along $z$ are normalized by $\lambda_{\infty}$, a quantity that is readily evaluated in DNS. DNS statistics are normalized by their averaged value in the far-field turbulence zone. In practical terms, this is defined as the region $3<z / \lambda_{\infty}<4.6$ in a frame of reference with $z=0$ coinciding with the $\mathrm{T} / \mathrm{NT}$ interface (the upper limit corresponding to the edge of the simulation domain). This procedure mitigates the effect of spurious oscillations in the DNS statistics due to an imperfect averaging process. The location of the T/NT interface is related between the RDT and the DNS using the value of $\overline{|\boldsymbol{\omega}|^{2}}$. At $t=0$, this quantity follows a Heaviside step function in the RDT model, with a discontinuity at $z=0$, but as time evolves it becomes positive at $z<0$ due to growth of the VBL. In the DNS data, a translation is performed on $z / \lambda_{\infty}$, so that the normalized values of $\overline{|\boldsymbol{\omega}|^{2}}$ are the same at a prescribed value of $z<0$ as in the RDT model. It must be stressed that this translation generally differs for each time considered. It is also worth noting that in the DNS the vorticity can propagate into the initially non-turbulent flow zone due to distortion of the T/NT interface and mixing of the turbulent and nonturbulent fluid. However, for the short times since boundary insertion considered here this effect should be negligible.

Results will be presented for two different times, corresponding to $\delta / \lambda_{\infty}=0.1$ and 0.2 . It was chosen to use $\delta / \lambda_{\infty}$ as a measure of the time, since this is the quantity that appears naturally in the RDT solutions. Since $\delta=2(\nu t)^{1 / 2}$, then

$$
\frac{\delta}{\lambda_{\infty}}=2 R e_{\lambda}^{-1 / 2}\left(\frac{q t}{\lambda_{\infty}}\right)^{1 / 2}
$$

Admitting, consistently with the assumptions of $\S 2$, that $t$ must be considerably smaller than $T_{L}$, the dimensionless time on the right hand side of (3.30) must be $q t / \lambda_{\infty} \ll 1$, and so, for $R e_{\lambda}=30$ (such as considered in the DNS - see $\S 2.3$ ), $\delta / \lambda_{\infty} \ll 0.36$. The values of $\delta / \lambda_{\infty}$ quoted above roughly satisfy this condition.

Figure 9 presents a comparison between results from RDT (lines) and from DNS (symbols) for $\delta / \lambda_{\infty}=0.1$. Figure $9(a-c)$ shows the velocity variances, figure $9(d)$ the TKE, figure $9(e)$ the dissipation rate and figure $9(f)$ the vorticity variance normalized by its value in the far-field turbulence $\omega_{\infty}^{2}=\overline{|\boldsymbol{\omega}|^{2}}(z \rightarrow+\infty)$. It can be seen that statistics which were discontinuous at $z=0$ in the inviscid case, for example $\overline{u^{2}} / q^{2}$ and $\overline{v^{2}} / q^{2}$ and $K / K_{\infty}$, are now continuous at the $\mathrm{T} / \mathrm{NT}$ interface, in order to satisfy the boundary conditions. The inviscid peaks that existed at $z=0^{+}$for these quantities are also smoothed considerably. The quantities that depend on the variances and covariances of the strain rates, like $\varepsilon / \varepsilon_{\infty}$ and $\overline{|\boldsymbol{\omega}|^{2}} / \omega_{\infty}^{2}$, have their discontinuities replaced by large maxima at $z=0$, mainly due to the contribution given by $\overline{(\partial u / \partial z)^{2}}$, which departs from an infinite initial value at $z=0$. Despite some oscillations in the DNS data, which suggest an incomplete statistical convergence of the averages, and a slight underestimation of $\overline{u^{2}} / q^{2}$ and $K / K_{\infty}$ slightly above $z=0$, the agreement between RDT and DNS is remarkably good for all quantities, even those that are sharply peaked. This confirms that the resolution employed in the DNS is sufficient for the present purposes (see detailed discussion in $\S 2.3$ ).

Figure 10(a) displays a comparison between $\lambda / \lambda_{\infty}$ from RDT and from DNS at $\delta / \lambda_{\infty}=0.1$. It may be seen that the jump that existed at $z=0$ in the inviscid RDT result (see figure 8 ) was smoothed. The DNS data are in very good agreement with RDT except in the region $z<0$, where the Taylor microscale is very sensitive to the relative decay rates of $\overline{u^{2}}$ and $\overline{(\partial u / \partial x)^{2}}$. Since this aspect is dependent on the form assumed for the energy spectrum of the turbulence, this behaviour is not surprising. The underesti- 

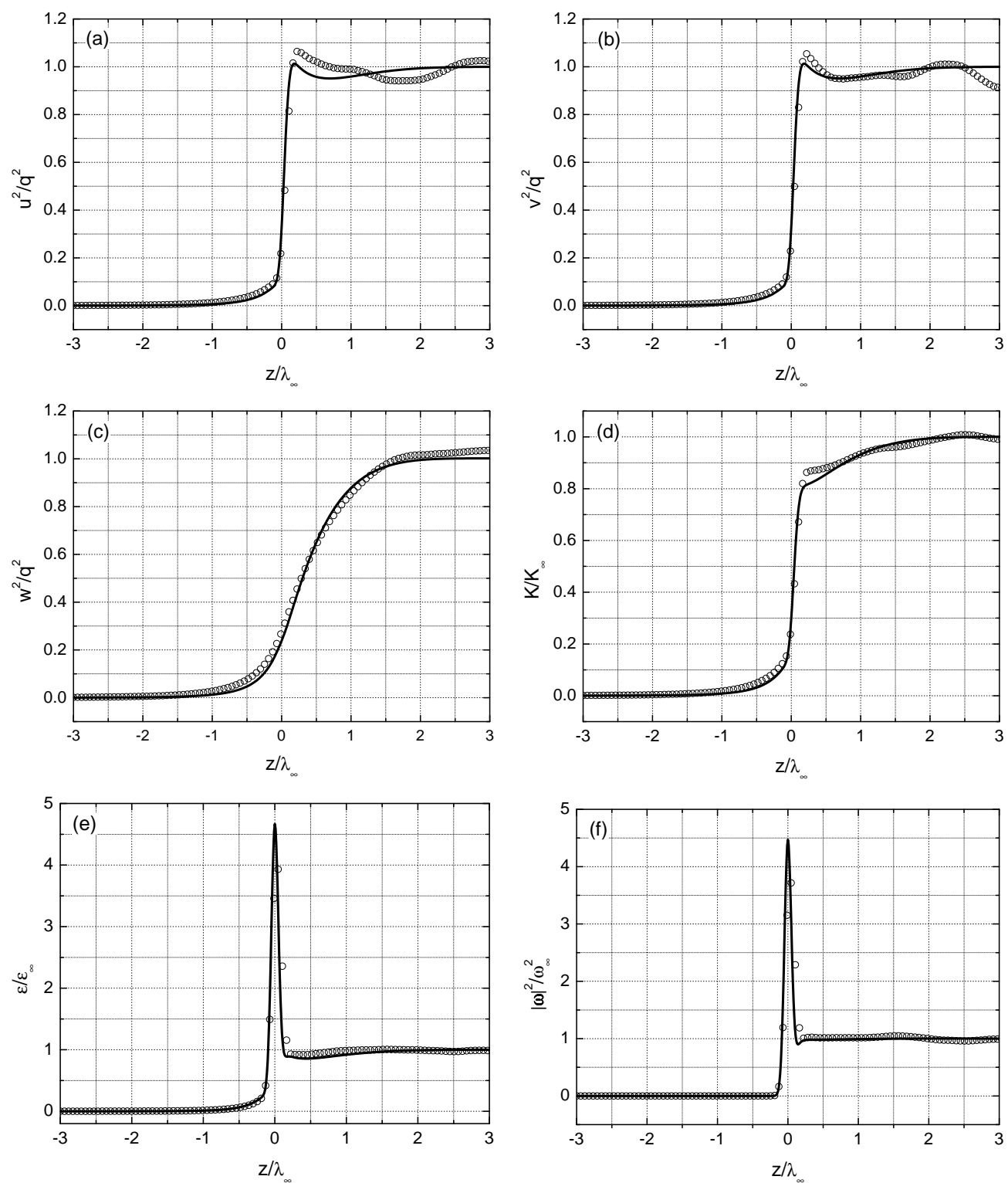

Figure 9. Flow statistics from viscous RDT (lines) and from DNS (symbols) for $\delta / \lambda_{\infty}=0.1$. (a) $\overline{u^{2}} / q^{2},(b) \overline{v^{2}} / q^{2},(c) \overline{w^{2}} / q^{2},(d) K / K_{\infty},(e) \varepsilon / \varepsilon_{\infty},(f) \overline{|\boldsymbol{\omega}|^{2}} / \omega_{\infty}^{2}$

mation of the DNS data by the RDT model means that not enough energy is present in the turbulence spectrum at wavenumbers inversely proportional to the distances where this underestimation occurs. Nevertheless, both RDT and DNS results exhibit a clear linear increase as $z \rightarrow-\infty$. In figure $10(b)$, the mean pressure, conventionally assumed to be zero far outside the turbulence, is shown. The RDT result presented is inviscid (similar to that of figure 5), since viscous effects influence very little the behaviour of the pressure. For that reason, the considerable effort necessary to derive a viscous RDT solution for $\bar{p} /\left(\rho q^{2}\right)$ was deemed not worthwhile. The agreement between RDT and DNS is remarkably good. This is even more striking if one takes into account the fact that $\bar{p}$ 

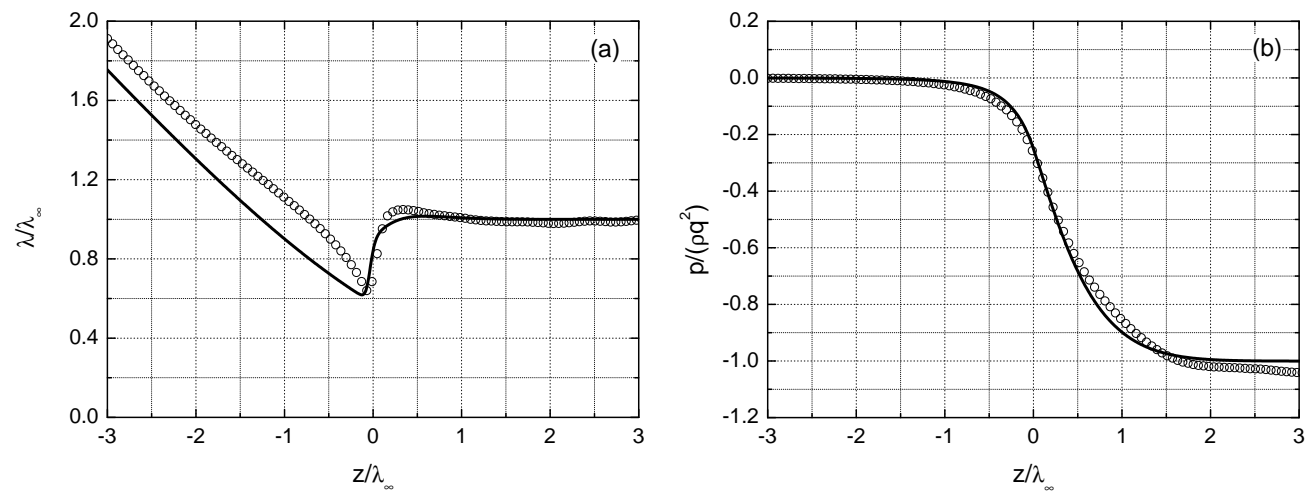

Figure 10. Flow statistics from RDT (lines) and from DNS (symbols) for $\delta / \lambda_{\infty}=0.1$. $(a)$ $\lambda / \lambda_{\infty},(b) p /\left(\rho q^{2}\right)$. Note that the latter quantity has been calculated using inviscid RDT.
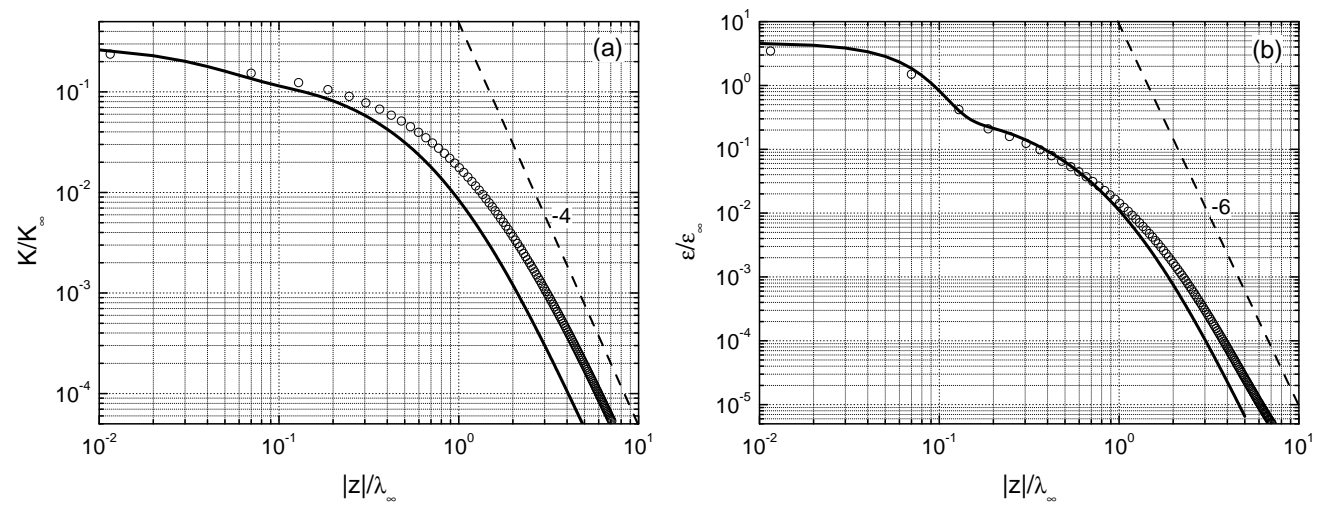

FiguRE 11. Flow statistics at $z<0$ normalized by their values at $z \rightarrow+\infty$ from viscous RDT (lines) and DNS (symbols) for $\delta / \lambda_{\infty}=0.1$. The dashed lines correspond to power laws with -4 and -6 exponents. (a) TKE, (b) dissipation rate.

was not normalized by its value at $z \rightarrow+\infty$ in the DNS results, but simply scaled using $q$, as is done in RDT.

In figure 11, the asymptotic behaviour of the TKE and of the dissipation rate is displayed in the irrotational flow zone, from RDT (lines) and from DNS (symbols) for $\delta / \lambda_{\infty}=0.1$. Despite a slight underestimation of both quantities by RDT (which may be related with the assumed form for the energy spectrum, for the same reasons as pointed out above for figure $10(a)$ ), the $z^{-4}$ and $z^{-6}$ power laws as $z \rightarrow-\infty$ are followed by the DNS data, as expected.

Figure 12 shows similar results as figure 9 , but at a later time for which $\delta / \lambda_{\infty}=0.2$. The maxima of $\overline{u^{2}} / q^{2}, \overline{v^{2}} / q^{2}, K / K_{\infty}$, and especially those of $\varepsilon / \varepsilon_{\infty}$ and $\overline{|\boldsymbol{\omega}|^{2}} / \omega_{\infty}^{2}$, are further decreased, and the agreement between RDT and DNS is a little worse than in figure 9. All DNS statistics in the irrotational flow zone are slightly underestimated by RDT. A larger underestimation than in figure 9 also occurs in the turbulent zone near the T/NT interface for $\overline{w^{2}} / q^{2}, \varepsilon / \varepsilon_{\infty}$ and $\overline{|\boldsymbol{\omega}|^{2}} / \omega_{\infty}^{2}$. Still, the agreement remains quite reasonable, in particular the peaks of $\varepsilon / \varepsilon_{\infty}$ and $\mid \boldsymbol{\omega}^{2} / \omega_{\infty}^{2}$ are accurately diagnosed. Since $\lambda / \lambda_{\infty}$ and $\bar{p}$ varied relatively little between $\delta / \lambda_{\infty}=0.1$ and $\delta / \lambda_{\infty}=0.2$, it was decided not to present profiles of these quantities at the later time.

As in inviscid conditions, closed analytical expressions for the turbulence statistics at 

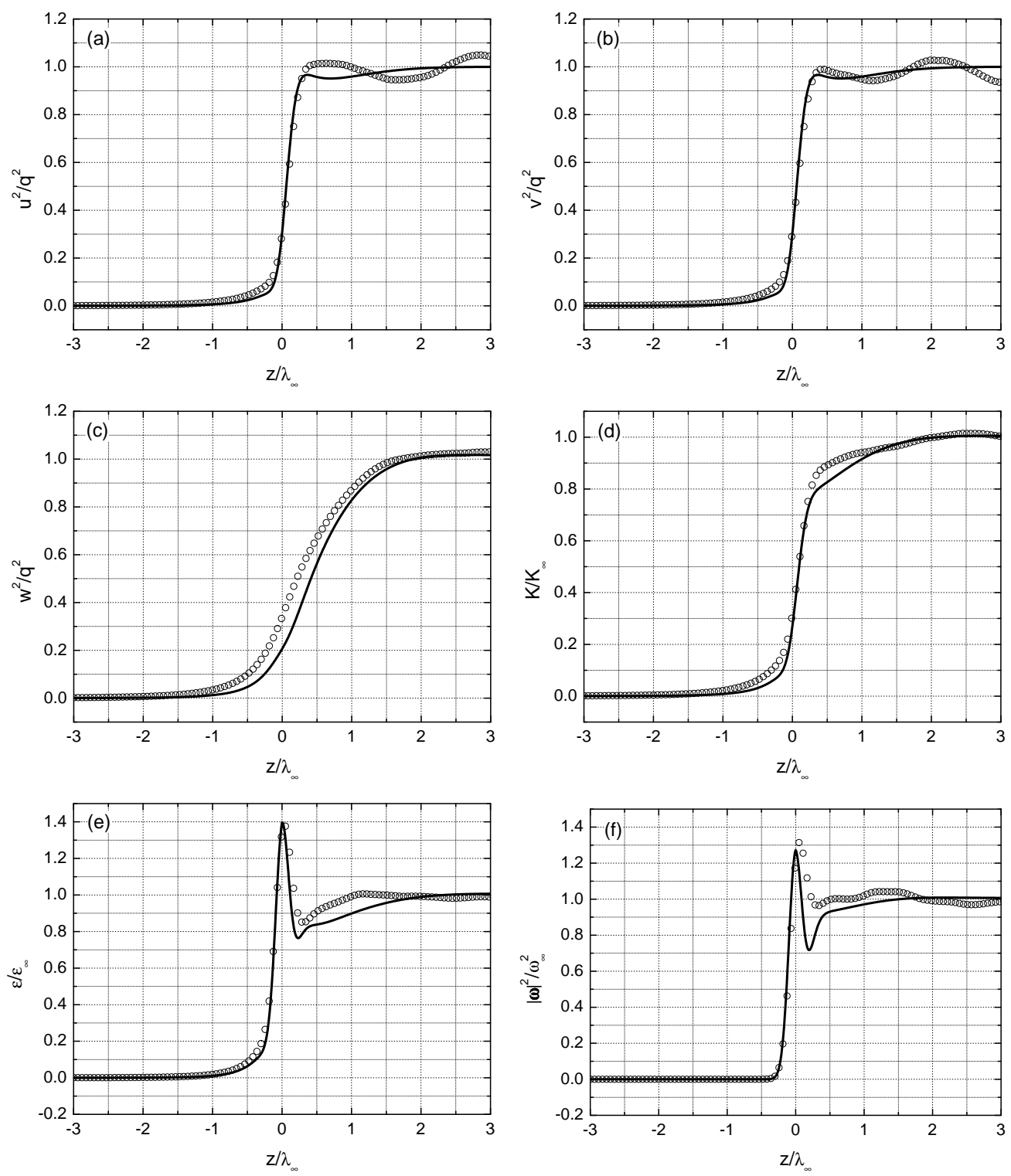

Figure 12 . The same as figure 9 , but for $\delta / \lambda_{\infty}=0.2$.

$z=0$ can be calculated from RDT, but depend now on the shape of the assumed energy spectrum. The TKE, for example, can be shown to be

$$
\frac{K(z=0)}{K_{\infty}}=\frac{1}{3}-\frac{\pi}{6 \sqrt{2}}\left(\frac{\delta}{\lambda_{\infty}}\right)+\left(\frac{5 \pi}{24}-\frac{5}{12}+\frac{1}{12 \pi}\right)\left(\frac{\delta}{\lambda_{\infty}}\right)^{2}+\frac{35}{48}\left(\frac{\delta}{\lambda_{\infty}}\right)^{4} .
$$

Although this expression is exactly consistent with the RDT solutions found previously, the second- and fourth-order terms are unreliable, since $u$ was only expanded up to first order in $\delta / \lambda_{\infty}$, and so additional second- and fourth-order terms are missing from (3.31). Moreover, the zeroth- and second-order terms are independent of the energy spectrum, 
because they result from the integrals (2.27). The same cannot be said about the firstand fourth-order terms.

The dissipation rate and the vorticity variance are given by analogous expressions:

$$
\begin{aligned}
\frac{\varepsilon(z=0)}{\varepsilon_{\infty}}= & \frac{2}{15 \pi}\left(\frac{\delta}{\lambda_{\infty}}\right)^{-2}+\frac{31}{60}+\frac{1}{15 \pi}-\frac{9 \pi}{15 \sqrt{2}}\left(\frac{\delta}{\lambda_{\infty}}\right) \\
& +\left(\frac{49 \pi}{80}-\frac{7}{20}+\frac{1}{15 \pi}\right)\left(\frac{\delta}{\lambda_{\infty}}\right)^{2}+\frac{21}{20}\left(\frac{\delta}{\lambda_{\infty}}\right)^{4}, \\
\frac{\overline{|\omega|^{2}}(z=0)}{\omega_{\infty}^{2}}= & \frac{2}{15 \pi}\left(\frac{\delta}{\lambda_{\infty}}\right)^{-2}+\frac{1}{4}-\frac{1}{15 \pi} \\
& +\left(\frac{7 \pi}{240}+\frac{1}{15 \pi}-\frac{7}{12}\right)\left(\frac{\delta}{\lambda_{\infty}}\right)^{2}+\frac{21}{20}\left(\frac{\delta}{\lambda_{\infty}}\right)^{4} .
\end{aligned}
$$

For similar reasons as given above for the TKE, only the terms of order lower than zero in (3.32) and (3.33) are reliable, and only the terms of order -2 and zero are independent of the form of the energy spectrum.

Equations (3.31), (3.32) and (3.33) relate the values of turbulence statistics at $z=0$ with the ratio $\delta / \lambda_{\infty}$. This is particularly important for $\varepsilon$ or $\overline{|\boldsymbol{\omega}|^{2}}$, since there is a peak in either quantity at the T/NT interface. The magnitude of this peak can thus be related to $\delta / \lambda_{\infty}$, which is useful for studying the scaling of $\delta$, as will be shown next.

\subsection{The scaling of $\delta$ at high Reynolds number}

Although many of the results presented in previous sections were independent of $R e_{\lambda}$, a relativaly low value of this parameter was used in the DNS and implied in RDT. In contrast, in the following scaling analysis a more realistic situation with high $R e_{\lambda}$ will be considered. By assuming similarity of high Reynolds number flows, the particular value taken by $R e_{\lambda}$ becomes irrelevant for this analysis. The only requirement is that $R e_{\lambda}$ be sufficiently high for the flow to present a clear scale separation between the Taylor and Kolmogorov microscales.

An interesting question arising in the context of $\mathrm{T} / \mathrm{NT}$ interfaces is the scaling for the VBL thickness $\delta$. Essentially, two possibilities have been identified: either $\delta \sim \lambda_{\infty}$ (in the present notation) or $\delta \sim \eta$, where $\eta$ is the Kolmogorov spatial microscale. Using turbulent plane jets (for $60<R e_{\lambda}<160$ ) and SFT (for $30<R e_{\lambda}<110$ ), da Silva \& Taveira (2010) have shown that the VBL thickness is equal to the vortex core radius of the biggest vortices existing near the $\mathrm{T} / \mathrm{NT}$ interface, $\delta \approx R$. Using the equilibrium Burgers vortex model, the radius of these structures is $R \sim\left(\nu / S^{\prime}\right)^{1 / 2}$, where $S^{\prime}$ is the norm of the local strain rate. Using this result, da Silva \& Taveira (2010) infer that $S^{\prime} \sim q / L_{\infty}$ in free shear layers, which leads to $\delta \approx R \sim\left(\nu / S^{\prime}\right)^{1 / 2} \sim \lambda_{\infty}$, whereas in SFT the strain is imposed by the background turbulence $-S^{\prime} \sim q / \lambda_{\infty}-$ yielding $\delta \sim \eta$. These scaling laws are in excellent agreement with DNS and experimental data for shear-driven turbulence and SFT for a wide range of Reynolds numbers (da Silva \& Taveira 2010; Westerweel et al. 2005, 2009).

It is tempting to see whether RDT is able to point to one of these two scaling laws, e.g. $\delta \sim \lambda_{\infty}$ or $\delta \sim \eta$. In light of the results described above, one would expect to get $\delta \sim \eta$, since we are dealing here with turbulence with zero mean shear. This is indeed what is observed in the present DNS (also used in da Silva \& Taveira (2010)), where the thickness of the vorticity jump at the T/NT interface is $\delta \approx 2.7 \eta$.

As we shall see below, RDT is able to suggest an answer for the scaling of the VBL, by assuming the existence of an equilibrium VBL thickness, corresponding to extrapolating 
RDT to near its limit of validity. The procedure adopted here is motivated by Teixeira \& Belcher (2000), and by several other previous linear studies, which suggest that the structure of turbulence at equilibrium (e.g., when the VBL attains its equilibrium thickness in forced turbulence) is reproduced qualitatively by RDT as long as the turbulence statistics are evaluated at $t=O\left(T_{L}\right)$.

The concept of an equilibrium thickness of the VBL merits some discussion. While entrainment may play an important role in the sharpening of the T/NT interface, opposing its viscous diffusion (Westerweel et al. 2009), it is also quite plausible that the same nonlinear processes that keep the VBL thin in flows near solid walls or free surfaces, such as those addressed by Perot \& Moin (1995), play an important, if not dominant role. The RDT estimates developed below explore that possibility. An equilibrium VBL thickness may not exist in decaying turbulence. But in that situation the other turbulence length scales, in particular the Taylor microscale, appear to increase with time at the same rate as the VBL thickness (Wang \& George 2002), so the same scalings as for forced turbulence probably apply. This gives additional relevance to the following analysis.

Before proceeding with our analysis, however, it is important to recall that the definition of the T/NT interface location used here is slightly different from the one used in other recent papers, for example Westerweel et al. (2009) and Bisset et al. (2002). Whereas in the present paper the $\mathrm{T} / \mathrm{NT}$ interface location corresponds to the maximum value of the vorticity norm, in e.g. da Silva \& Taveira (2010) the T/NT interface is defined as the surface where the vorticity norm is higher than a given threshold, where the threshold corresponds to the smaller value of the vorticity norm that does not change the magnitude of the conditional vorticity inside the turbulent region (details can be found in Bisset et al. (2002)). Even though the procedure used here to detect the T/NT interface is slightly different, and could serve as a basis for an alternative method to detect the $\mathrm{T} / \mathrm{NT}$ interface, arguably it should lead to similar results concerning the thickness of the VBL.

Bearing in mind all these issues, an RDT estimate for the thickness of an equilibrium VBL can be derived from the definition of $\delta$ at $t=T_{L}$, i.e.

$$
\delta \sim\left(\nu T_{L}\right)^{1 / 2} \Rightarrow \frac{\delta}{\lambda_{\infty}} \sim R e_{\lambda}^{-1 / 2}\left(\frac{q T_{L}}{\lambda_{\infty}}\right)^{1 / 2} .
$$

If, as is usual in RDT studies (including Teixeira \& Belcher 2000), $T_{L}$ is assumed to scale like $L_{\infty} / q$, then

$$
\frac{q T_{L}}{\lambda_{\infty}} \sim \frac{L_{\infty}}{\lambda_{\infty}} \sim R e_{\lambda} \varepsilon^{\prime}
$$

where $\varepsilon^{\prime}=\varepsilon L_{\infty} / q^{3}$ is the dimensionless dissipation rate. Since $\varepsilon^{\prime}$ is known to be approximately constant and of $O(1)$ at relatively high $R e_{\lambda}$ (Burattini, Lavoie \& Antonia 2005; Ishihara, Gotoh \& Kaneda 2009), subject to these conditions (3.34) and (3.35) yield

$$
\frac{\delta}{\lambda_{\infty}} \sim \varepsilon^{1 / 2} \sim O(1) \quad \Rightarrow \quad \delta \sim \lambda_{\infty}
$$

which is the scaling obtained by da Silva \& Taveira (2010) for shear-driven turbulence.

If, however, $T_{L}$ is assumed to scale like $\lambda_{\infty} / q$, as was found to be required for the formal validity of the present RDT model, then from (3.34)

$$
\frac{\delta}{\lambda_{\infty}} \sim R e_{\lambda}^{-1 / 2} \quad \text { or, since } \quad \frac{\eta}{\lambda_{\infty}} \sim R e_{\lambda}^{-1 / 2} \text { then } \delta \sim \eta
$$

and the scaling inferred by da Silva \& Taveira (2010) for SFT is recovered. 
It is interesting to see that the new results derived here based on RDT are consistent with the two possibilities identified by da Silva \& Taveira (2010). i.e. $\delta \sim \lambda_{\infty}$ (in shear-driven turbulence) and $\delta \sim \eta$ (in SFT). Recall that those authors do not discard the possibility of having $\delta \sim \eta$ even in shear-driven turbulence, for instance when the Reynolds number is very high and the larger eddies are fragmented, in which case the radius of the surviving vortices may be closer to the Kolmogorov than to the Taylor microscale. However, the interesting point here is that RDT arguments do not totally exclude the possibility of having $\delta \sim \lambda_{\infty}$ even when there is no mean shear, something that was not initially contemplated by da Silva \& Taveira (2010).

The foregoing analysis shows that the scaling for $\delta$ (3.36) or (3.37) provided by RDT depends on the scaling for $T_{L}$, the time that limits the validity of this theory. Generally speaking, the definition of $T_{L}$ based on the integral length scale is used with the argument that RDT is a model only applicable to the large eddies, and the spatial length scale of these eddies is $L_{\infty}$. However, one must recognize that the correct scaling for the nonlinear terms neglected in RDT (e.g. $(\boldsymbol{u} \cdot \boldsymbol{\nabla}) \boldsymbol{u})$ involves $\lambda_{\infty}$ rather than $L_{\infty}$, because this is the spatial scale of the velocity gradients in the turbulence (cf. (3.27)). It must be stressed that use of this last scaling unifies the two requirements for the validity of RDT invoked in the abstract of Teixeira \& Belcher (2000), as it leads to a condition equivalent to their Equations (4.27) or (4.28). This, along with the fact that (3.37) agrees with the previous arguments by da Silva \& Taveira (2010), favours the idea that this last scaling is the correct one, i.e. RDT arguments also imply that $\delta \sim \eta$.

\section{Concluding remarks}

RDT was used to explicitly calculate statistics characterizing the flow structure near an interface separating SFT from an irrotational flow region. The viscous coupling between these two regions was fully taken into account, extending the calculations of Carruthers \& Hunt (1986), and revisiting the problem first addressed by the pioneering study of Phillips (1955). The T/NT interface was assumed to be instantaneously introduced, and to remain essentially flat during the process of adjustment between the two regions of the flow (though mass transport across the interface is permitted). This problem is relevant for understanding the physical processes controlling turbulent entrainment across $\mathrm{T} / \mathrm{NT}$ interfaces, and may help the development of subgrid-scale models for LES specifically suited to those regions, since it has been shown recently that the classical LES approach is deficient in the vicinity of T/NT interfaces (da Silva 2009).

The behaviour of the inviscid RDT solutions was first explored. Profiles of the velocity variances, TKE, strain-rate covariances, viscous dissipation rate, vorticity variance and various turbulence length scales were calculated across the T/NT interface. The values of these statistics adjacent to each side of the $\mathrm{T} / \mathrm{NT}$ interface (where they often are discontinuous) normalized by their corresponding values in the bulk of the turbulence, were calculated, and found to be independent of the form of the turbulence energy spectrum.

In agreement with previous DNS results (da Silva \& Pereira 2008), the viscous dissipation rate, unlike the vorticity variance, was found to be non-zero in the irrotational flow zone, but instead to decay to zero proportionally to $z^{-6}$ at relatively large distances from the T/NT interface. This result parallels the analogous asymptotic dependency first derived by Phillips (1955) for the velocity variances (or equivalently, the TKE).

The mean pressure distribution associated with the velocity fluctuations of the flow was calculated for the first time using RDT. It was found that, as one moves from the irrotational into the turbulent flow zone, the mean pressure progressively decreases, displaying a maximum of its gradient slightly inside the turbulent zone. In a more realistic 
model, this pressure distribution would perhaps induce a mean flow into the turbulence, i.e. an entrainment velocity.

The turbulence integral length scales and the Taylor microscale showed a linear increase away from the $\mathrm{T} / \mathrm{NT}$ interface in the irrotational flow region, but the dissipation length scale $K^{3 / 2} / \varepsilon$ attains a constant asymptotic value in the same region. A turbulent diffusivity defined in terms of the TKE and $\varepsilon$ decays to zero proportionally to $z^{-2}$.

The viscous RDT solutions were then compared with the results of DNS. In the DNS, the idealized situation considered in RDT was reproduced, viz. a T/NT interface was suddenly introduced at the initial time. All of the statistics alluded to above in an inviscid context showed a very good agreement with DNS data in the viscous case at two different times, corresponding to $\delta / \lambda_{\infty}=0.1,0.2$. As expected, in quantities like the velocity variances, the TKE, or the integral length scales, inviscid discontinuities existing initially are progressively smoothed by viscous effects as time advances, while the viscous dissipation rate and the vorticity variance display large maxima at the $\mathrm{T} / \mathrm{NT}$ interface, which decay with time and can be related analytically to $\delta / \lambda_{\infty}$.

The present results provide a solid basis for the development of subgrid-scale closures designed to cope with the formidable challenges posed by the region near the T/NT interface. Moreover, the results can be used to devise a new, more accurate, method to define and detect the exact location of the T/NT interface, and to test the scaling for its thickness, using DNS or experimental data. Finally, the scaling $\delta \sim \eta$ obtained by da Silva \& Taveira (2010) for SFT, together with the scaling arguments derived here from RDT, seem to imply that the time limit for the validity of RDT is $\lambda_{\infty} / q$ rather than $L_{\infty} / q$, at least for the particular type of flow under consideration here, i.e. SFT.

The authors acknowledge the financial support of the Portuguese Foundation for Science and Technology (FCT) under Projects PEst-OE/CTE/LA0019/2011-IDL and PTDC/EME-MFE/099636/2008.

\section{Appendix. Definition of the matrices $M_{i j}^{ \pm}$}

The matrices $M_{i j}^{ \pm}$relate the Fourier transforms of the velocity field at any point with the Fourier transforms of the homogeneous and isotropic turbulent velocity assumed to exist at $z \rightarrow+\infty$, in accordance with (2.21). The non-zero elements of these matrices are defined as follows:

$$
\begin{aligned}
& M_{11}^{-}=M_{22}^{-}=\frac{1}{2}\left(1+\frac{1}{2} \mathrm{i} \sqrt{\pi} \delta k_{3}\right)\left[\operatorname{erf}\left(\frac{z}{\delta}\right)+1\right], \\
& M_{13}^{-}=\frac{\mathrm{i} k_{1}}{2 k_{12}} \mathrm{e}^{k_{12} z}-\frac{1}{4} \mathrm{i} \sqrt{\pi} \delta k_{1}\left[\operatorname{erf}\left(\frac{z}{\delta}\right)+1\right], \\
& M_{23}^{-}=\frac{\mathrm{i} k_{2}}{2 k_{12}} \mathrm{e}^{k_{12} z}-\frac{1}{4} \mathrm{i} \sqrt{\pi} \delta k_{2}\left[\operatorname{erf}\left(\frac{z}{\delta}\right)+1\right], \\
& M_{33}^{-}=\frac{1}{2}\left[\mathrm{e}^{k_{12} z}+\delta\left(\mathrm{i} k_{3}-\frac{1}{2} \sqrt{\pi} \delta k^{2}\right)\left\{\frac{z}{\delta}\left[\operatorname{erf}\left(\frac{z}{\delta}\right)+1\right]+\frac{\mathrm{e}^{-z^{2} / \delta^{2}}}{\sqrt{\pi}}\right\}\right], \quad(z<0), \\
& M_{11}^{+}=M_{22}^{+}=\mathrm{e}^{\mathrm{i} k_{3} z}+\frac{1}{2}\left(1-\frac{1}{2} \mathrm{i} \sqrt{\pi} \delta k_{3}\right)\left[\operatorname{erf}\left(\frac{z}{\delta}\right)-1\right], \\
& M_{13}^{+}=\frac{\mathrm{i} k_{1}}{2 k_{12}} \mathrm{e}^{-k_{12} z}+\frac{1}{4} \mathrm{i} \sqrt{\pi} \delta k_{1}\left[\operatorname{erf}\left(\frac{z}{\delta}\right)-1\right],
\end{aligned}
$$




$$
\begin{aligned}
M_{23}^{+}= & \frac{\mathrm{i} k_{2}}{2 k_{12}} \mathrm{e}^{-k_{12} z}+\frac{1}{4} \mathrm{i} \sqrt{\pi} \delta k_{2}\left[\operatorname{erf}\left(\frac{z}{\delta}\right)-1\right], \\
M_{33}^{+}= & \mathrm{e}^{\mathrm{i} k_{3} z}-\frac{1}{2} \mathrm{e}^{-k_{12} z}-\frac{1}{2} \delta^{2} k^{2} \\
& +\frac{1}{2} \delta\left(\mathrm{i} k_{3}+\frac{1}{2} \sqrt{\pi} \delta k^{2}\right)\left\{\frac{z}{\delta}\left[\operatorname{erf}\left(\frac{z}{\delta}\right)-1\right]+\frac{\mathrm{e}^{-z^{2} / \delta^{2}}}{\sqrt{\pi}}\right\}, \quad(z>0) .
\end{aligned}
$$

The remaining elements of $M_{i j}^{ \pm}$are equal to zero.

\section{REFERENCES}

Alvelius, K. 1999 Random forcing of three-dimensional homogeneous turbulence. Phys. Fluids 11(7), 1880-1889.

Batchelor, G. K. \& Proudman, I. 1954 The effect of rapid distortion of a fluid in turbulent motion. Q. J. Mech. Appl. Maths. 7, 83-103.

Bisset, D. K., Hunt, J. C. R. \& Rogers, M. M. 2002 The turbulent/non-turbulent interface bounding a far wake. J. Fluid Mech. 451, 383-410.

Burattini, P., Lavoie, P. \& Antonia, R. A. 2005 On the normalized turbulent energy dissipation rate. Phys. Fluids 17, 098103.

Carruthers, D. J. \& Hunt, J. C. R. 1986 Velocity fluctuations near an interface between a turbulent region and a stably stratified layer. J. Fluid Mech. 165, 475-501.

Corrsin, S. \& Kistler, A. L. 1955 Free-stream boundaries of turbulent flows. Technical Report TN-1244. NACA.

Fernando, H. J. S. \& Hunt, J. C. R. 1997 Turbulence, waves and mixing at shear-free density interfaces. 1. a theoretical model. J. Fluid Mech. 347, 197-234.

Hunt, J. C. R. 1984 Turbulence structure in thermal convection and shear-free boundary layers. J. Fluid Mech. 138, 161-184.

Hunt, J. C. R., Eames, I. \& Westerweel, J. 2006 Mechanics of inhomogeneous turbulence and interfacial layers. J. Fluid Mech. 554, 499-519.

Hunt, J. C. R. \& Graham, J. M. R. 1978 Free stream turbulence near plane boundaries. J. Fluid Mech. 84, 209-235.

Hunt, J. C. R., Stretch, D. D. \& Belcher, S. E. 2011 Viscous coupling of shear-free turbulence across nearly flat fluit interfaces. J. Fluid Mech. 671, 96-120.

Ishinara, T., Gotoh, T. \& KAnEDA, Y. 2009 Study of high-reynolds number isotropic turbulence by direct numerical simulation. Annu. Rev. Fluid Mech. 41, 165-180.

JimÉNEZ, J. \& WRAY, A. 1998 On the characteristics of vortex filaments in isotropic turbulence. J. Fluid Mech. 373, 255-285.

Perot, B. \& Moin, P. 1995 Shear-free turbulent boundary layers. part 1. physical insights into near-wall turbulence. J. Fluid Mech. 295, 199-227.

Phillips, O. M. 1955 The irrotational motion outside a free turbulent boundary. Proc. Camb. Phil. Soc. 51, 220-229.

DA Silva, C. B. 2009 The behaviour of subgrid-scale models near the turbulent/nonturbulent interface in jets. Phys. Fluids 21, 081702.

DA Silva, C. B. \& Pereira, J. C. F. 2008 Invariants of the velocity-gradient, rate-of-strain, and rate-of-rotation tensors across the turbulent/nonturbulent interface in jets. Phys. Fluids 20, 055101.

DA Silva, C. B. \& Reis, R. N. 2011 The role of coherent vortices near the turbulent/nonturbulent interface in a planar jet. Phil. Trans. Roy. Soc. A 369, 738-753.

DA Silva, C. B. \& Taveira, R. R. 2010 The thickness of the $\mathrm{t} / \mathrm{nt}$ interface is equal to the radius of the large vorticity structures near the edge of the shear layer. Phys. Fluids 22, 121702 .

Teixeira, M. A. C. \& Belcher, S. E. 2000 Dissipation of shear-free turbulence near boundaries. J. Fluid Mech. 422, 167-191.

Teixeira, M. A. C. \& Miranda, P. M. A. 1997 On the entrainment in schatzmann's integral plume model. Appl. Sci. Res. 57, 15-42. 
Tennekes, H. \& Lumley, J. L. 1972 A first course in turbulence. The MIT Press.

Tordella, D., Iovieno, M. \& BAiley, P. R. 2008 Sufficient condition for gaussian departure in turbulence. Phys. Review E 77, 016309.

Townsend, A. A. 1966 The mechanism of entrainment in free turbulent flows. J. Fluid Mech. 26, 689-715.

Townsend, A. A. 1976 The Structure of Turbulent Shear Flow. Cambridge University Press.

Turner, J. S. 1986 Turbulent entrainment: the development of the entrainment assumption, and its application to geophysical flows. J. Fluid Mech. 173, 431-471.

WANG, H. \& GeORGe, W. K. 2002 The integral scale in homogeneous isotropic turbulence. $J$. Fluid Mech. 459, 429-443.

Westerweel, J., Fukushima, C., Pedersen, J. M. \& Hunt, J. C. R. 2005 Mechanics of the turbulent-nonturbulent interface of a jet. Phys. Review Lett. 95, 174501.

Westerweel, J., Fukushima, C., Pedersen, J. M. \& Hunt, J. C. R. 2009 Momentum and scalar transport at the turbulent/non-turbulent interface of a jet. J. Fluid Mech.631, 199-230. 